\title{
Maturation of Spontaneous Firing Properties after Hearing Onset in Rat Auditory Nerve Fibers: Spontaneous Rates, Refractoriness, and Interfiber Correlations
}

\author{
익ingjing Sherry Wu, ${ }^{1}{ }^{-}$Eric D. Young, ${ }^{2}$ and Elisabeth Glowatzki ${ }^{1,3}$ \\ ${ }^{1}$ Department of Neuroscience, ${ }^{2}$ Department of Biomedical Engineering, and ${ }^{3}$ Department of Otolaryngology-Head and Neck Surgery, Center for Hearing \\ and Balance and the Center for Sensory Biology, Johns Hopkins University School of Medicine, Baltimore, Maryland 21205
}

Auditory nerve fibers (ANFs) exhibit a range of spontaneous firing rates (SRs) that are inversely correlated with threshold for sounds. To probe the underlying mechanisms and time course of $S R$ differentiation during cochlear maturation, loose-patch extracellular recordings were made from ANF dendrites using acutely excised rat cochlear preparations of different ages after hearing onset. Diversification of SRs occurred mostly between the second and the third postnatal week. Statistical properties of ANF spike trains showed developmental changes that approach adult-like features in older preparations. Comparison with intracellularly recorded EPSCs revealed that most properties of ANF spike trains derive from the characteristics of presynaptic transmitter release. Pharmacological tests and waveform analysis showed that endogenous firing produces some fraction of ANF spikes, accounting for their unusual properties; the endogenous firing diminishes gradually during maturation. Paired recordings showed that ANFs contacting the same inner hair cell could have different SRs, with no correlation in their spike timing.

Key words: auditory nerve fiber; development; hair cell; intrinsic firing; refractoriness; spontaneous rate

\section{Significance Statement}

The inner hair cell (IHC)/auditory nerve fiber (ANF) synapse is the first synapse of the auditory pathway. Remarkably, each IHC is the sole partner of 10-30 ANFs with a range of spontaneous firing rates (SRs). Low and high SR ANFs respond to sound differently, and both are important for encoding sound information across varying acoustical environments. Here we demonstrate SR diversification after hearing onset by afferent recordings in acutely excised rat cochlear preparations. We describe developmental changes in spike train statistics and endogenous firing in immature ANFs. Dual afferent recordings provide the first direct evidence that fibers with different SRs contact the same IHCs and do not show correlated spike timing at rest. These results lay the groundwork for understanding the differential sensitivity of ANFs to acoustic trauma.

\section{Introduction}

Auditory nerve fibers (ANFs) exhibit spontaneous activity in the absence of external sound (Kiang et al., 1965). In vivo studies have shown that ANFs have various spontaneous rates (SRs) that are in-

\footnotetext{
Received April 6, 2016; revised Aug. 17, 2016; accepted Aug. 23, 2016.

Author contributions: E.D.Y. and E.G. designed research; I.S.W. performed research; J.S.W. and E.D.Y. analyzed data; J.S.W., E.D.Y., and E.G. wrote the paper.

This work was supported by National Institute on Deafness and Other Communication Disorders Grants R01DC006476 and R01DC012957 to E.G. and National Institute on Deafness and Other Communication Disorders P30 DC005211 to the Center for Hearing and Balance at Johns Hopkins. We thank Tessa Ropp for cat ANF data in Figures $2 B$ and $4 E$; Eunyoung Yi and Lisa Grant for recordings that were included in the analysis of EPSCS and AP waveforms; and Lisa Grant for initiating the project on simultaneous recordings of two fibers at one hair cell in the laboratory. The authors declare no competing financial interests.

Correspondence should be addressed to Dr. Elisabeth Glowatzki, Johns Hopkins School of Medicine, Department of Otolaryngology-Head and Neck Surgery, Center for Hearing and Balance, 720 Rutland Avenue, Ross 824, Baltimore, MD 21205. E-mail: eglowat1@jhmi.edu.

DOI:10.1523/JNEUROSCI.1187-16.2016

Copyright $\odot 2016$ the authors $\quad 0270-6474 / 16 / 3610584-14 \$ 15.00 / 0$
}

versely correlated with the acoustic threshold and dynamic range of the fiber (Sachs and Abbas, 1974; Liberman, 1978). High SR fibers are more sensitive (lower acoustic thresholds) and have a narrower dynamic range than do low SR fibers, which have higher thresholds and a nonsaturating stimulus-response curve. This diversity of properties allows different groups of ANFs to facilitate hearing in varied acoustic environments. In addition, ANFs are differentially sensitive to acoustic trauma, with low SR ANFs being more sensitive to noise-induced synaptic neuropathy (Furman et al., 2013). To fully understand stimulus encoding and neuropathy, it is important to understand the development of the diverse SR distribution of ANFs as well as the properties of the spike trains.

Several altricious species (e.g., cat, mouse, and rat) are born deaf, and their auditory system matures rapidly in early postnatal life, making them good animal models for studying auditory system development and SR maturation. In kittens, the developmental diversification of SRs and the appearance of high SR ANFs 
happen over several weeks postnatally (Walsh and McGee, 1987). However, it is not clear when exactly SRs diversify in rat. In vitro recordings from ANF terminals in mice and rats provide an important approach for investigating the underlying mechanisms of ANF firing with controlled pharmacological manipulation (Glowatzki and Fuchs, 2002; Grant et al., 2010; Rutherford et al., 2012). To follow the development of SR and the properties of ANF spike trains, loose-patch extracellular recordings from ANF terminals were performed in vitro on acutely excised rat cochlear coils at three ages after hearing onset, covering weeks $2-5$ postnatally. The results were compared with in vivo data from adult animals.

Statistical analysis of spike trains recorded in vitro showed that, with age, the range of SRs increased, the proportion of regular-firing patterns declined, and spontaneous activity increasingly showed the features observed in mature ANFs. Furthermore, statistical properties of presynaptic release, inferred from intracellularly recorded EPSCs, showed most of the properties of in vivo ANF spontaneous spike trains, including irregular discharge and long-term autocorrelation; this result suggests a presynaptic origin of these properties. Pharmacological tests provided evidence for some endogenous firing in immature ANFs, which contributes to the atypical spike-train properties of developing ANFs. Finally, loose-patch recordings from pairs of ANFs contacting the same inner hair cell (IHC) confirmed the hypothesis that low and high SR fibers contact the same IHC (Liberman, 1982). Moreover, the spike trains of fibers contacting the same IHC were uncorrelated, showing that individual presynaptic release sites behave independently at rest.

\section{Materials and Methods}

Animals and preparations. In accordance with animal protocols approved by the Johns Hopkins University Animal Care and Use Committee, rats of either sex at postnatal days (P) 15-32 (Sprague Dawley; Charles River; RGD catalog \#734476, RRID:RGD_734476) were deeply anesthetized by isoflurane inhalation and decapitated. Cochleae were removed from the temporal bone and placed in extracellular solution (see below). The apical cochlear coil was excised and pinned on a coverslip. The coverslip was transferred to the recording chamber under an upright microscope (Axioskop2 FS plus, Zeiss). The preparation was visualized using a $40 \times$ water-immersion differential interference contrast objective with $4 \times$ magnification through a NC70 Newvicon camera (Dage MTI).

Data acquisition. In the recording chamber, extracellular solution was perfused at a rate of $\sim 0.7 \mathrm{ml} / \mathrm{min}$. Recordings were performed at room temperature $\left(22^{\circ} \mathrm{C}-25^{\circ} \mathrm{C}\right)$, except for one experimental series in which bath temperature was raised to $\sim 30^{\circ} \mathrm{C}$ using a Thermoclamp 1 Temperature Controller (Automate Scientific). Recording pipettes were fabricated from $1 \mathrm{~mm}$ borosilicate glass (WPI). Pipettes were pulled with a multistep horizontal puller (Sutter Instruments), coated with Sylgard (Dow Corning), and fire polished. Pipette resistances were 11-15 $\mathrm{M} \Omega$ for intracellular recordings and $8-13 \mathrm{M} \Omega$ for loose-patch recordings. Data were acquired using pCLAMP 9.2 software (Molecular Devices) in conjunction with a Multiclamp 700A amplifier (Molecular Devices). The signal was low-pass filtered at $10 \mathrm{kHz}$ and digitized at 20 or $50 \mathrm{kHz}$ with a Digidata 1322A (Molecular Devices).

For voltage-clamp intracellular recordings of ANF bouton endings, holding voltages (corrected for measured liquid junction potentials) were between -84 and $-99 \mathrm{mV}$. All recordings had a leak current $<200$ $\mathrm{pA}$, expect for one recording that had a leak current up to $500 \mathrm{pA}$. The series resistance (Rs) error was kept $<60 \mathrm{~m} \Omega$, and recordings were not corrected for Rs. Some data from in vivo recordings of spontaneous activity in cat ANFs were used for comparison purposes (T. Ropp and E.D.Y., unpublished data). Methods for these recordings (see Figs. 2B, $4 E$ ) were identical to those described by Heinz and Young (2004).

For loose-patch extracellular recordings of ANF bouton endings, recordings were obtained when the seal resistance reached $\sim 2-5$ times the pipette resistance, at $\sim 30-80 \mathrm{M} \Omega$. Because of the high density of ANF innervation at the bottom of the IHC, spikes from more than one ANF may be visible in a recording. In most of these cases, spikes from different fibers could be clearly separated from each other based on the different amplitudes. Recordings were excluded from the study if unambiguous separation of the two spike trains in a recording was not possible. Because the presence of events is the sole determinant of a successful recording, this method inherently undersampled fibers with very low SR.

Chemicals and solutions. Extracellular solutions for loose-patch extracellular and voltage-clamp intracellular recordings contained the following (in mM): $5.8 \mathrm{KCl}, 144 \mathrm{NaCl}, 0.9 \mathrm{MgCl}_{2}, 1.3 \mathrm{CaCl}_{2}, 0.7 \mathrm{NaH}_{2} \mathrm{PO}_{4}, 5.6$ glucose, 10 HEPES, pH $7.4(\mathrm{NaOH}), 300$ mOsm. Pipette solutions for intracellular voltage-clamp recordings contained the following (in $\mathrm{mM}$ ): $20 \mathrm{KCl}, 110 \mathrm{~K}$-methanesulfonate, $5 \mathrm{MgCl}_{2}, 0.1 \mathrm{CaCl}_{2}, 5$ EGTA, 5 HEPES, $5 \mathrm{Na}_{2} \mathrm{ATP}, 0.3 \mathrm{Na}_{2} \mathrm{GTP}, 5 \mathrm{Na}_{2}$ phoshocreatine, pH $7.2(\mathrm{KOH}), 290$ mOsm or $135 \mathrm{KCl}, 3.5 \mathrm{MgCl}_{2}, 0.1 \mathrm{CaCl}_{2}, 5$ EGTA, 5 HEPES, 0-4 $\mathrm{Na}_{2} \mathrm{ATP}, 0-0.2 \mathrm{Na}_{2} \mathrm{GTP}$, pH $7.2(\mathrm{KOH}), 290$ mOsm. TTX citrate, NBQX disodium salt, $(R S)$ - $\alpha$-methyl-4-carboxyphenylglycine, CNQX disodium salt, and $(R S)$-CPP were purchased from R\&D Systems. Cadmium chloride $\left(\mathrm{CdCl}_{2}\right)$ was purchased from Sigma-Aldrich. Drug application was mediated by whole-bath perfusion or a gravity-driven flow pipette (100- $\mu$ m-diameter opening) placed near the row of IHCs and connected with a VC-6 channel valve controller (Warner Instruments).

Event detection and graphing. Data were analyzed and plotted using Clampfit (Molecular Devices), Origin 9.1 (OriginLab), MiniAnalysis (Synaptosoft; RRID:SCR_014441), MATLAB (The MathWorks), and Illustrator (Adobe). Extracellular spikes were detected using a routine in MiniAnalysis, with a threshold set at $\sim 3$ times the value of the root mean square of the baseline noise and subsequently accepted by eye.

For intracellular EPSCs, events were monopolar negative current pulses with a rapid onset $(<0.3 \mathrm{~ms})$ and a smooth, approximately exponential decay (time constant of $\sim 0.5 \mathrm{~ms}$ ), as for the monophasic EPSC in Figure $1 F$ (second event). These events were detected as for extracellular events and were marked as occurring at the peak of the pulse. However, there were also multiphasic EPSCs (see Fig. $1 F$, first and third events), and we assume that these are the superposition of two or more release events (Glowatzki and Fuchs, 2002; Li et al., 2009). Individual events were separated out of a multiphasic cluster by marking an event at each rapid onset in the waveform, timed at the peak (see Fig. $1 F$, red X's). In addition to manual checking of the event detection results in MiniAnalysis, the analysis was repeated by deconvolving the raw EPSC data with a kernel function consisting of the average of isolated monophasic events. This process gives a narrow peak for each event that corresponds well with the events marked as described above.

Segments of recordings shown in the figures of this paper were filtered with a high-pass Bessel 8-pole filter at $5 \mathrm{~Hz}$, and, in some cases, an additional low-pass Gaussian filter at $10 \mathrm{kHz}$. For some recordings, the data points were reduced by decimation to a $10 \mathrm{kHz}$ sampling rate. The average waveform was the average of individual events aligned by the peak and scaled to the same amplitudes. The first derivative of the average waveform was smoothed with a Savitzky-Golay filter using a 5 point quadratic approximation.

Spike and EPSC train analysis. Interval histograms were computed and plotted as the un-normalized number of interevent intervals (IEIs, the events may be either extracellular spikes or EPSCs) in $1 \mathrm{~ms}$ bins (larger bins were used in low-rate spike trains; smaller bins were used to reveal brief features, such as some peaks). A straight line was fit by least-squares to the logarithm of the IEI histogram (i.e., the log of the IEI counts) between $10 \%$ and $90 \%$ of the interval on the right edge of the IEI histogram at which the spike count in a bin first reached 0 . This line was used to compare the histogram with a simple exponential decay. Hazard functions were computed as the ratio of the number of IEIs in a particular bin to the sum of the number of events in that bin and all later bins; the hazard is thus an estimate of the probability of an event at a particular interval, given that no event has yet occurred. The hazard functions were normalized by the bin width, to yield estimates of instantaneous rate as a function of time since the previous event. The absolute value of the slope of the line fit to the IEI histogram (described above) is the "hazard rate," 
which is the value expected in the hazard function if the decay of the IEI histogram is exponential.

The Poisson point process was used as a null comparison model for the data. A Poisson process has a constant probability of event occurrence per unit time, and the probability of an event occurrence is statistically independent of the timing of previous events (Cox and Lewis, 1978). For neurons, a more realistic null model has a short absolute refractory period (ARP) immediately after a spike, during which the probability of a spike is zero and no spikes occur. Following the ARP, spiking may be assumed to occur at a constant rate, as in a Poisson process (Young and Barta, 1986; Teich, 1992; Heil et al., 2007). However, in more sophisticated (and accurate) models, the instantaneous rate changes slowly after the end of the ARP (Miller and Mark, 1992; Kumar and Johnson, 1993). Idealized Poisson spiking is characterized by an exponential IEI histogram, a constant hazard function, and zero serial correlation between successive IEIs. Real ANF spike trains have an ARP, a slow rise in instantaneous discharge rate following the ARP (Gaumond et al., 1982; Li and Young, 1993; Heil et al., 2007), and (usually) negative correlation of successive IEIs and positive correlation of intervals separated by at least one (Lowen and Teich, 1992; Prijs et al., 1993; Peterson et al., 2014).

The Fano factor was used to characterize spiking regularity. Event (spike or EPSC) times were collected into successive bins of duration $T$. The mean $\mu$ and variance $\sigma^{2}$ of the counts were computed from all the bins, and the Fano factor is the ratio $\sigma^{2} / \mu$. Fano factor was computed at series of logarithmically equally spaced bin widths $T$, from $1 \mathrm{~ms}$ to a maximum value at which there are only 10 bins (e.g., for a 600 s recording, the maximum $T$ would be $60 \mathrm{~s}$ ). In ANFs, there is a power-law relationship between Fano factor and $T$ for larger values of $T$, which is produced by rate fluctuations in the spike train (Teich, 1992; Kumar and Johnson, 1993). This is called the "fractal" property of ANF spiking. To measure the extent of the fractal property, the fractal dimension was estimated as the slope of a best-fitting line through a doublelogarithmic Fano factor time curve (log Fano factor vs $\log T$ ) using all the points for which the Fano factor was $>1.1$; sometimes it was necessary to adjust this number manually to make sure the line was fit to the power-law range of $T$ values.

As a comparison for the Fano factor time curve, the calculation was repeated after randomly rearranging (shuffling the order of) the IEIs in the event train and then constructing an event train with the shuffled intervals; the Fano factor calculation was then repeated with the shuffled event train. The shuffling was repeated 10 times and the resulting Fano factor time curve averaged arithmetically to compute the final comparison. This shuffling process preserves the overall average rate and the IEI statistics (IEI histogram and hazard function) of the event train but destroys any long-term relationships among event times, such as the rate fluctuations mentioned above and correlations among intervals. Thus, a shuffled train is expected not to show the fractal property in Fano factor time curve (Teich, 1992; Kumar and Johnson, 1993; Kelly et al., 1996).

To evaluate the degree of coordinated firing between two fibers recorded simultaneously, a cross-correlogram was computed as the number of spike coincidences in $1 \mathrm{~ms}$ bins separated by a lag varying from -200 to $200 \mathrm{~ms}$. The number of coincidences was normalized by dividing by the bin width and the number of events in one of the two trains. This allows the histogram to be interpreted as the average spike rate in the second spike train at various delays (lags) from spikes in the first train. The selection of the normalizing spike train affects only the average rate, and not the shape of the cross-correlogram, which reflects around zero lag (i.e., reverses on the time axis) when the normalizing spike train is changed.

Statistics. Statistical tests were performed with R version 3.1.2 (R Core Team, 2014) or with MATLAB (The MathWorks). For statistical analyses, data were tested using the Shapiro-Wilk normality test. Appropriate parametric or nonparametric tests were applied accordingly. Where two parameters were compared, $t$ tests, Wilcoxon rank-sum, or KolmogorovSmirnov tests were used. Where more than two parameters were compared, ANOVA, the $k$-sample permutation test (Hothorn et al., 2008; Fay and Shaw, 2010), or the Kruskal-Wallis test were used. To test the correlation between groups of data, Kendall's rank correlation tau was used. The level of statistical significance was $p<0.05$.

\section{Results}

Extracellular and intracellular recordings of spontaneous activity at ANF endings in vitro

To monitor the timing of action potential (AP) firing in ANFs in vitro, loose-patch extracellular recordings were performed at the unmyelinated bouton endings of ANFs close to where they contact the IHCs. Recordings were performed in acutely excised apical cochlear coils from rats, at P15-P32, an age range closely following the onset of hearing ( $\sim$ P12 for rats) (Jewett and Romano, 1972; Uziel et al., 1981; Ehret, 1983; Blatchley et al., 1987; Geal-Dor et al., 1993). The extracellular recording configuration was chosen over intracellular recordings to better preserve the structural integrity and intracellular environment of the postsynaptic endings and thereby to provide the most faithful representation of spike timing in individual fibers. For single-unit recordings from ANFs in vivo, spontaneous activity is defined as the activity that can be recorded without a sound signal present (Kiang et al., 1965). For the in vitro study here, the hair cell membrane potential was assumed to be "at rest" with $5.8 \mathrm{~mm} \mathrm{~K}^{+}$ and $1.3 \mathrm{mM} \mathrm{Ca}^{2+}$ in the extracellular solution. Under these conditions, APs in ANFs were considered "spontaneous".

A representative loose-patch recording from an ANF with spontaneous firing is shown in Figure $1 A$. In 14 of 14 recordings, spike-like events were blocked by TTX $(2-5 \mu \mathrm{M})$, suggesting that they represented APs (Fig. 1A). In some recordings, smaller events remained after TTX block as in this example. These TTXinsensitive events were blocked by the AMPA receptor blocker NBQX (20 $\mu \mathrm{M} ; n=1$; Fig. $1 B)$ and were thereby identified as EPSPs. Comparison of event sizes before and after TTX application (Fig. 1C,D) showed that APs and EPSPs could be well discriminated by amplitude. AP waveforms often, but not always, showed an inflection during depolarization (Fig. 1C,E, arrowheads), indicating that these APs were activated by an EPSP. As shown for extracellular recordings in other experimental settings (Brooks and Eccles, 1947; Fatt, 1957; Freygang and Frank, 1959; Terzuolo and Araki, 1961; Buzsáki et al., 1996; Henze et al., 2000), the waveform of the extracellularly recorded AP matched quite well with the first derivative of the intracellularly recorded AP waveform (Fig. 1E, right).

These data show that APs can be identified in loose-patch extracellular recordings from ANF endings and can be used to monitor spontaneous activity of ANFs in vitro.

\section{Spike trains and trains of EPSCs show fractal character in vitro}

To assess the characteristics of spontaneous activity in vitro, spike trains were studied in stable recordings that lasted $\geq 30 \mathrm{~min}(n=$ $5)$. Interestingly, rates often fluctuated slowly, on a scale of seconds to minutes, reminiscent of extracellular ANF recordings from anesthetized cats, a phenomenon that leads to "fractal" behavior (Teich et al., 1990). One of the features of fractal behavior is the similar appearance of rate-versus-time plots at progressively larger time scales. To illustrate this, spike counts were plotted using two different bin widths while keeping the same number of bins (Fig. 2A1,A2). Fluctuations in the SRs of ANFs did not decrease appreciably, even with 10-fold longer averaging time, the opposite of what is expected for a Poisson or a renewal counting process (Cox and Lewis, 1978). After shuffling (randomly reassigning) the order of the IEIs, which removes slow rate fluctuations and the associated long-term correlations between rates at different times, but preserves the relative frequencies of IEIs in the spike train, the fluctuations in rate were noticeably reduced in larger bins (Fig. 2A3, compare with Fig. 2A1). 
A

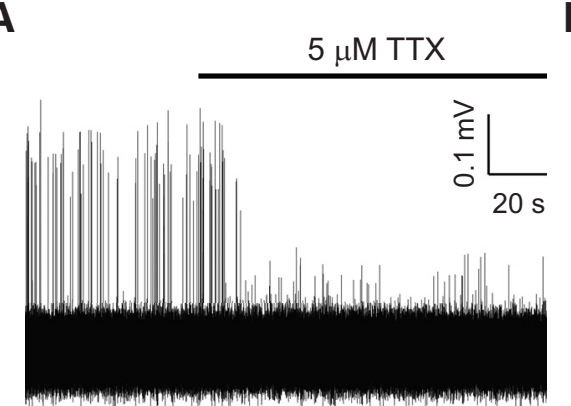

B

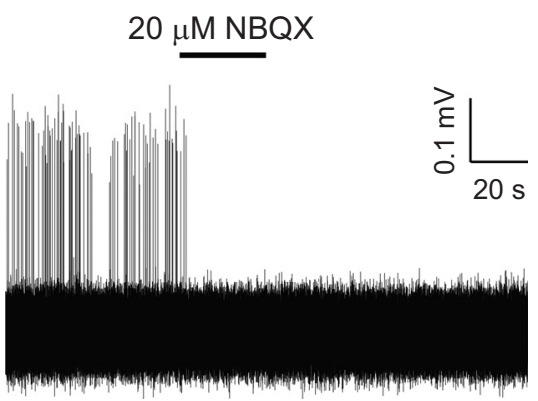

C

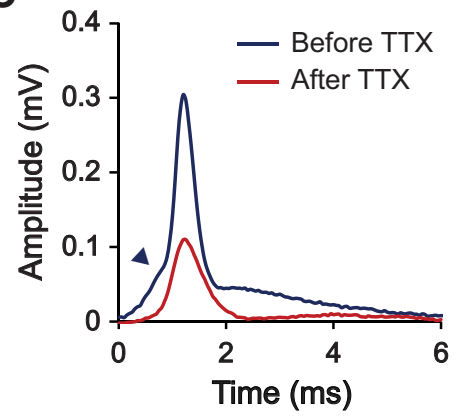

D

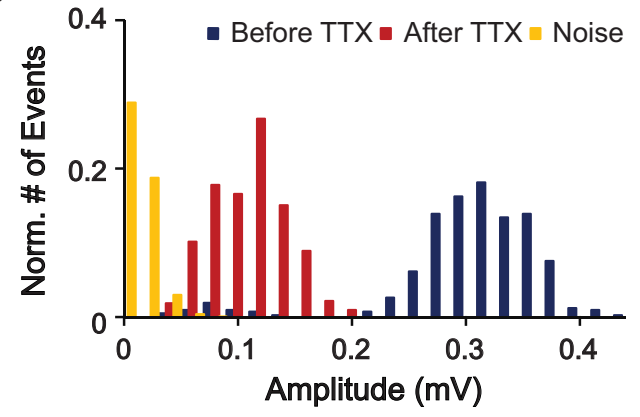

E

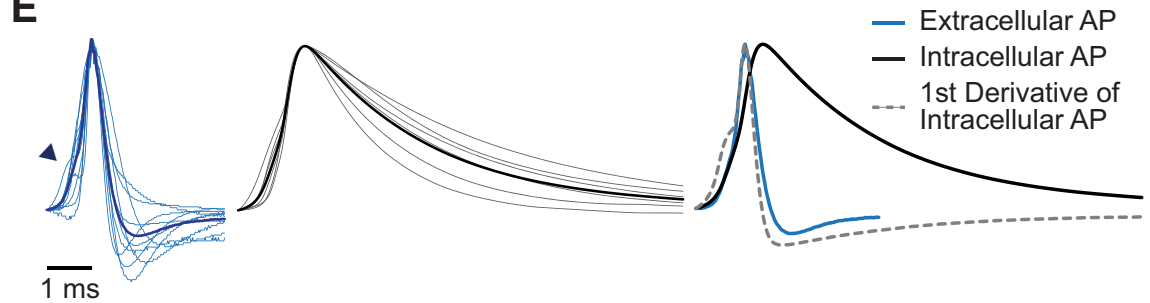

$\mathbf{F}$

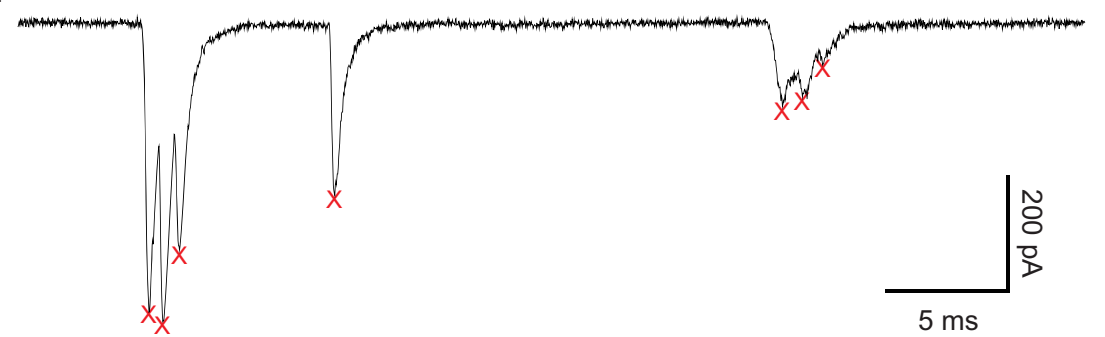

Figure 1. APs can be unambiguously identified in loose-patch extracellular recordings. $\boldsymbol{A}, \boldsymbol{B}$, Representative traces of a loosepatch extracellular recording from an ANF ending. Spikes in the recording were blocked by TTX $(\boldsymbol{A})$. Remaining smaller events were blocked by NBQX in the same fiber (applied previously and washed out), and thereby identified as EPSPs ( $\boldsymbol{B}$ ). $\boldsymbol{C}$, Average waveform of spikes in $\boldsymbol{A}$ shows inflection in the rising phase (arrowhead). $\boldsymbol{D}$, Amplitude histogram with normalized numbers of APs (blue), EPSPs (red), and noise (yellow) showing a clear separation of these three groups. E, Superimposed extracellularly recorded spikes from 10 ANFs (left in light blue), intracellularly recorded spikes from 6 ANFs (middle in gray), and their average waveforms (left in dark blue and middle in black). At right, the average waveforms are compared, along with the scaled first derivative of the intracellular waveform. Note the similarity of the first derivative with the extracellular waveform. $\boldsymbol{F}$, Whole-cell voltage-clamp recording from an ANF bouton showing typical spontaneous EPSCs. Two EPSCs are "multiphasic" with several peaks and one is "monophasic," with a single peak. Detected event times are marked with red X's. Holding potential was $-99 \mathrm{mV}$.

Another way to illustrate the fractal property is by plotting the Fano factor time curve, which is the ratio of the variance $\left(\sigma^{2}\right)$ to the mean $(\mu)$ number of spikes in bins of different widths, plotted against the bin width (Fig. 2A4). For a Poisson point process, $\sigma^{2}$ equals $\mu$, and the Fano factor should be equal to 1 for any bin width (dashed line). However, similar to results found in cat ANFs in vivo (Fig. 2B) (T. Ropp and E.D.Y., unpublished data), the Fano factor of in vitro spike trains was $<1$ over a range of small bin widths, a phenomenon known to be caused by refractory effects in ANFs (Young and Barta, 1986; Teich, 1992; Peterson et al., 2014), and then increased in a power-law fashion for bin widths $\sim>1 \mathrm{~s}$, suggesting the presence of fractal behavior (Fig. 2A4,C). The power-law dependence of Fano factor on bin width has been shown in simulation and modeling studies (Teich, 1992; Kumar and Johnson, 1993; Kelly et al., 1996) to be caused by the rate fluctuations discussed in the previous paragraph. The slow nature of the fluctuations leads to long-term correlation in spike rate and IEIs, which causes the increase in Fano factor. Shuffling the IEIs resulted in a loss of the correlation as well as the power-law increase of the Fano factor (Fig. $2 A 4, B, C$, green curves), confirming that the fractal property of the ANF spike trains arises from the serial order of the IEIs. At low SRs (Fig. 2C), the shuffled data often showed a small elevation of Fano factor above 1 for bin widths $>\sim 1 \mathrm{~s}$ and a small increasing trend. This elevation occurred because the low-SR spike train contains periods of no spiking, which increase the variability of the spike counts for larger bin widths; shuffling has no effect on this source of variability.

The slope of the power-law portion of the Fano factor time curve, called the fractal dimension, is a convenient measure of the fractal effect. All 5 ANFs analyzed exhibited fractal behavior with fractal dimension ranging from 0.66 to 0.82 , which lies within the range of $0.3-$ 0.9 , reported for spontaneous activity of cat ANFs in vivo (Teich, 1989). For fibers with "immature" spike train characteristics, which will be explained later (see Fig. 4), additional features sometimes appeared in the Fano factor time curve, but the power-law increase of Fano factor for larger bin widths still held. The refractory effect in the Fano factor time curve was smaller for spike trains with low SR, where the IEI was long compared with the refractory period, as shown in Figure $2 C$ (7 spikes/s vs 44 spikes/s in Fig. 2A4). This is the expected behavior on theoretical grounds (Young and Barta, 1986; Teich, 1992; Peterson et al., 2014) and is also seen in vivo.

To better localize the source of the fractal behavior, the timing of the presynaptic release events was estimated using the peak time of EPSCs in voltage-clamp intracellular recordings $(n=4, \mathrm{P} 17, \mathrm{P} 20, \mathrm{P} 27$, and P31 with release rates of 22,2 , 29 , and $67 /$ s, respectively). EPSCs presented with one or with multiple peaks (Fig. $1 F$ ), and every peak was counted based on the hypothesis that every peak represents a release event (see Materials and Methods). All Fano factor time curves from 


\section{Action Potentials}

A1

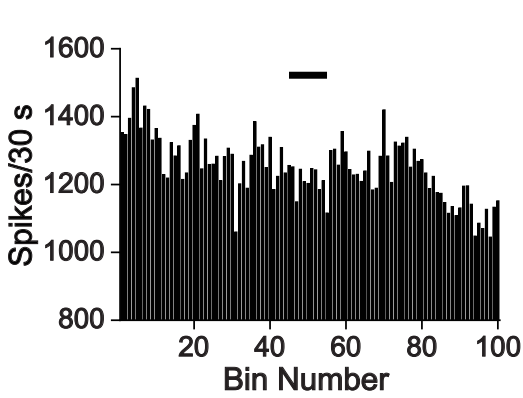

A3

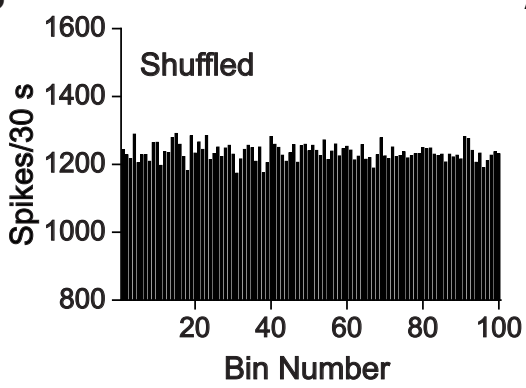

B

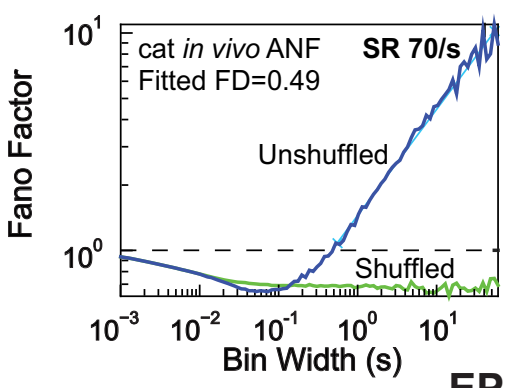

C

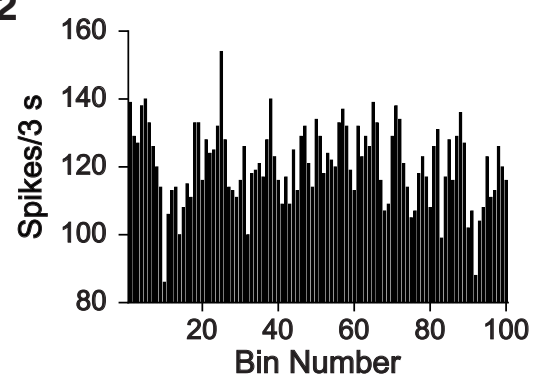

A4
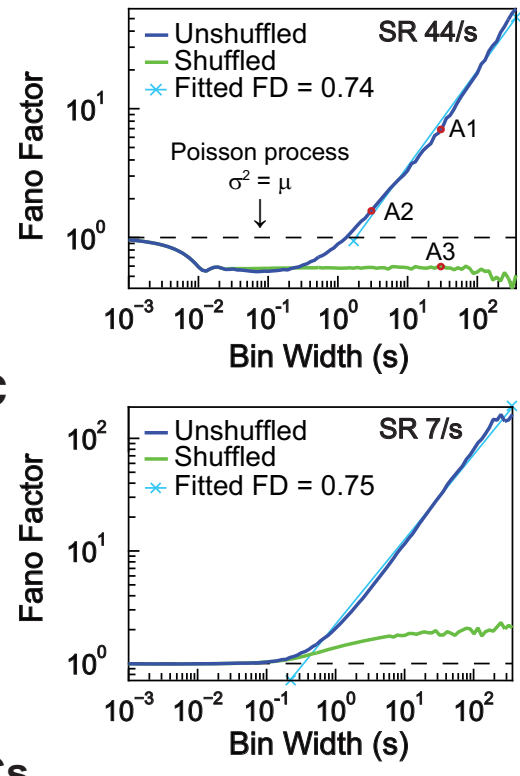

D1

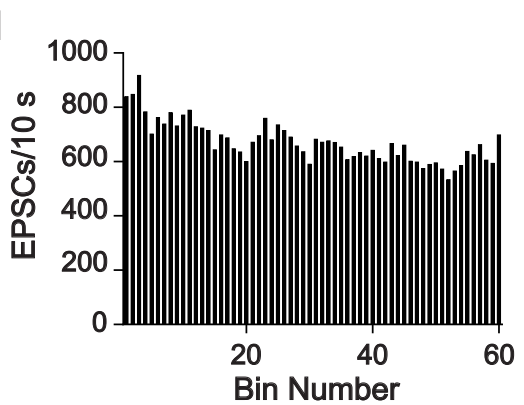

E1

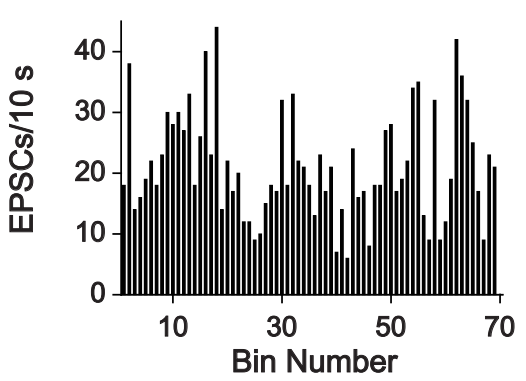

D2

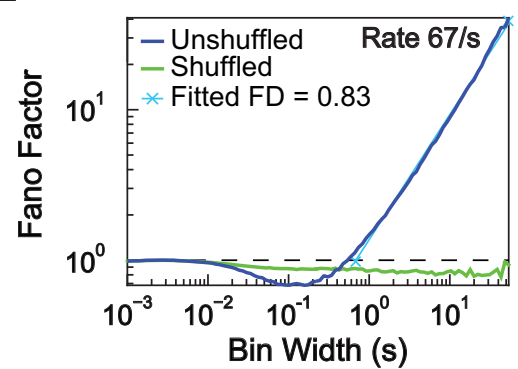

E2

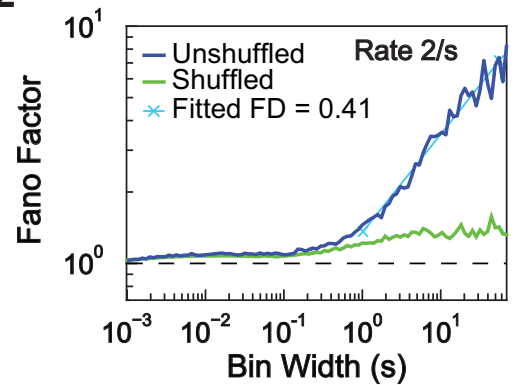

EPSCs showed the power-law behavior in the Fano factor time curves (Fig. $2 D, E)$, suggesting that presynaptic release mechanisms contribute to this fractal aspect of ANF spike trains. Shuffling the order of IEIs also eliminated the power-law increase of the Fano factor at larger bin widths for EPSCs. The effects of low discharge rate on the Fano factor of shuffled data (Fig. 2E2) were the same for EPSCs as for spikes (Fig. 2C), namely, a Fano factor near 1 for small bin widths and $>1$ (but little or no power-law rise) for large bin widths. For EPSCs with a high release rate, the Fano factor could be less than one over a range of smaller bin widths $(<1 \mathrm{~s}$; Fig. 2D2). For ANF firing, this dip in the Fano curve below 1 is attributed to refractoriness, as discussed previously. However, it will be shown in Figure 4 that EPSCs do not have a refractory period. Simulations showed that the Fanofactor dip in the EPSC data can be caused by event clustering in the multiphasic EPSCs; that is, either refractoriness or event clustering near $0 \mathrm{~ms}$ IEI can cause a similar dip.

The slow fluctuations in SR (Fig. 2A1-A3) that underlie the fractal behavior also produced positive serial correlation among the sequence of IEIs, meaning that long intervals tended to be followed by long intervals, and short intervals by short intervals, as the rate increased or decreased (data not shown). However, for ANF spike trains, the positive correlation occurs only between intervals separated by two or more spikes and not for successive intervals (which share a spike at the end of the first and beginning of the second interval); instead, successive intervals are (usually but not always) negatively correlated (Lowen and Teich, 1992; Prijs et al., 1993), which has been modeled as resulting from synaptic vesicle depletion and replenishment (Peterson et al., 2014). This pattern of negative and positive serial correlation also occurred in 3 of 4 EPSC recordings (SRs of 22, 29, and 67 spikes/s), but negative correlation was not observed in 1 of 4 EPSC recordings (SR of 2 spikes/s).
Figure 2. Extracellular spike trains and intracellular EPSCs recorded in vitro show fractal behavior. $\boldsymbol{A 1}$, $\boldsymbol{A 2}$, Rate plots from one ANF showing fluctuations of SR at different bin sizes (30 and 3 s). $\boldsymbol{A}$, Black bar represents the region covered by the abscissa in $\boldsymbol{A} \mathbf{2}$ $\mathbf{A 3}$, Rate plot of the same recording after shuffling the order of IEls; bin size is the same as $\boldsymbol{A} \mathbf{1}$. Note the reduced fluctuation of rate. A4, Fano factor time curve for the same recording, showing a power-law increase in Fano factor at longer bin widths (navy blue) and the loss of this increase when the order of the IEls was shuffled (green). FD, Fractal dimension (slope of the cyan line on $\log -\log$ coordinates). $\boldsymbol{A 4}$, The bin width for $\boldsymbol{A 1}, \boldsymbol{A} \mathbf{2}$, and $\boldsymbol{A} \mathbf{3}$ are marked (red circles). $\boldsymbol{B}$, Fano factor time curve of an in vivo cat ANF recording, for comparison (T. Ropp and E.D.Y., unpublished data). C, A second example of an in vitro recording from an ANF terminal with lower SR. D1, E1, Rate plots of EPSC counts from two representative whole-cell voltage-clamp recordings, showing rate fluctuations. Bin width is 10 s. D2, E2, Fano factor time curves of the same voltage-clamp recordings showing the same fractal features in presynaptic release as in spike trains. 
A

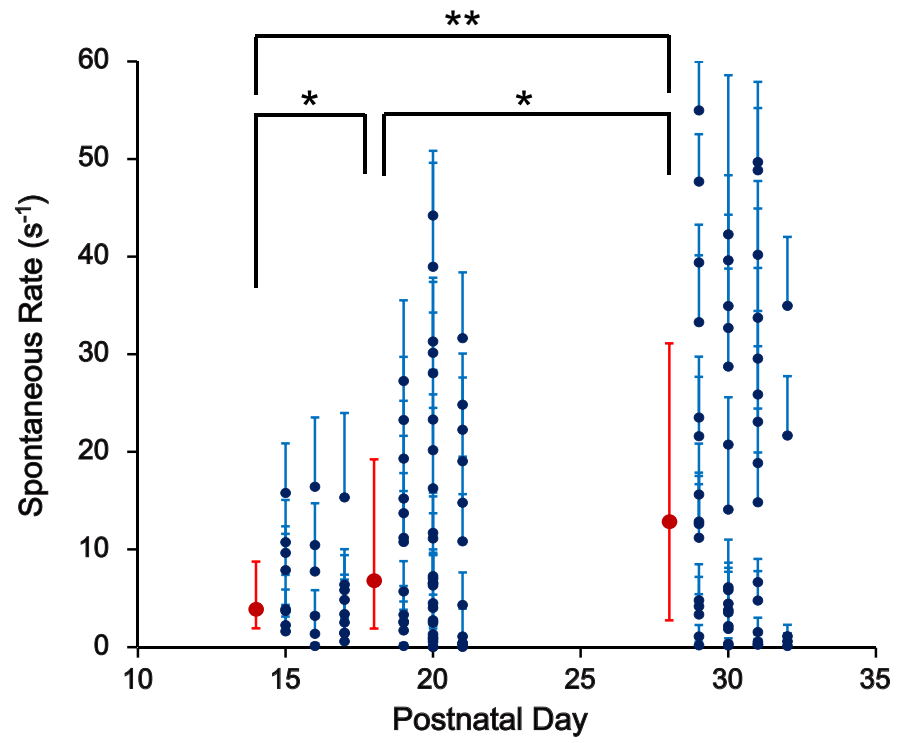

B

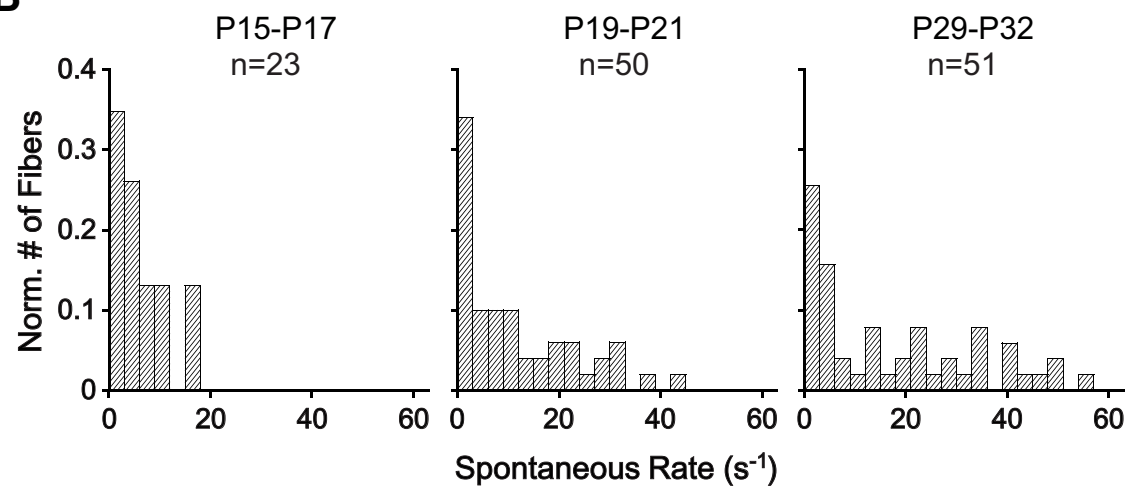

C

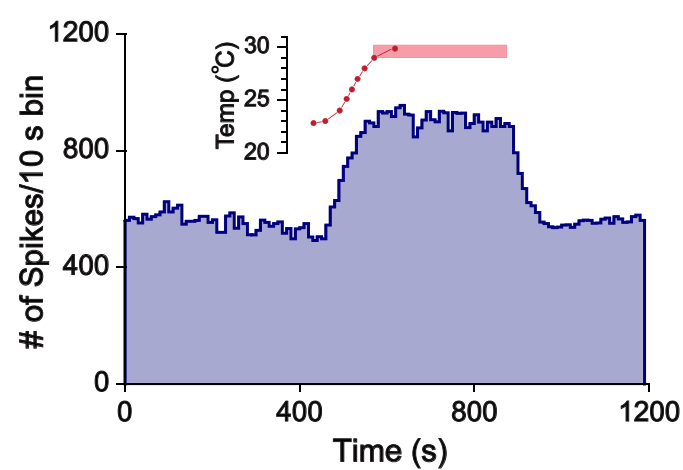

D

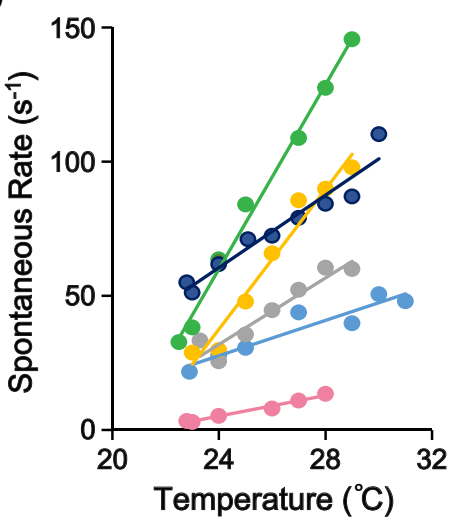

Figure 3. Diversification of SRs after hearing onset in ANFs recorded in vitro. $\boldsymbol{A}$, Plot of SRs of ANFs recorded at different ages. Navy blue dots represent the mean SR for an individual ANF. Cyan error bars indicate SEs with bin size of $1 \mathrm{~s}$. Red symbols represent the median rates. Red error bars indicate the first and third quartiles of each age group. Median SR for P15-P17 $(n=23)$, P19-P21 $(n=50)$, and P29-P32 ( $n=51)$ were 3.87, 6.80, and 12.85 spikes/s, respectively. Threesample permutation test was used to compare medians due to the skewed distribution of values. The median SR was significantly higher at P29-P32 compared with P19-P21 ( $p=0.04)$, als0 at P19-P21 compared with P15-P17 $(p=0.03)$, and P29-P32 compared with P15-P17 $(p=0.003)$. $\boldsymbol{B}$, Normalized histograms of SRs for ANFs recorded at P15-P17, $P 19-P 21$, and P29-P32. Bin size is 3 spikes/s. C, SR (blue) during one experiment in which bath temperature (red) was varied. SR increased when the bath temperature was increased and stayed at the elevated rate when the bath temperature stabilized at $29^{\circ} \mathrm{C}-30^{\circ} \mathrm{C}$ (pink bar). SR reversed to control values with restoration of the $23^{\circ} \mathrm{C}$ bath in this case. D, SR versus temperature for 6 such experiments during the temperature increase only. The navy blue fiber is the same unit as in $\boldsymbol{C}$. Note the wide range of slopes of the linear fits for the data points $\left(1.93-17.15\right.$ spikes $\left./ \mathrm{s}^{\circ} \mathrm{C}\right)$. ${ }^{*} p<0.05$. ${ }^{* *} p<0.01$.
In summary, the spontaneous activity of ANFs and EPSCs recorded in vitro is not stationary but exhibits fractal properties over longer time scales, similar to what has been found for ANFs in vivo.

\section{Diversification of SRs after hearing onset}

In vivo, in adult rats (4-12 months old), SRs of ANFs have been reported to range from 0 to 120 spikes/s (el Barbary, 1991), whereas before hearing onset (P0-P9), SRs were found to be $<5$ spikes/s at room temperature in vitro (Tritsch and Bergles, 2010). It is unknown when exactly during development the SRs of rat ANFs diversify. Experiments were performed to test whether diverse SRs can be measured in vitro, and if so, to chart the time course of diversification of SRs during rat cochlear development.

Because of the fractal properties of ANF spike trains, the SR varied during recordings (Fig. 2A1,A2). The following standard procedure was adopted to compute a representative SR. The first $2 \mathrm{~min}$ of the recording was excluded to allow time for the recording to stabilize. Thereafter, a minimum of $5 \mathrm{~min}$ and a maximum of 10 min of recording time were analyzed. For some low SR fibers $(n=7)$, a longer recording time was chosen to reach a minimum of 2000 spikes.

Three ages were chosen to study the development of SRs: the beginning and the end of postnatal week 3 (P15-P17 and $\mathrm{P} 19-\mathrm{P} 21)$ and the beginning of postnatal week 5 (P29-P32) (Fig. 3A). At P15-P17, the SR ranged from 0.10 to 16.42 spikes/s ( $n=23$; median $=3.87$ spikes $/ \mathrm{s})$. Only a few days later, at P19-P21, the SR was significantly higher (for statistics, see legend to Fig. 3), ranging from 0 to 44.22 spikes/s $(n=50$; median $=6.80$ spikes $/ \mathrm{s})$. SR covered an even larger range at P29-P32 from 0.11 to 54.99 spikes/s $(n=51$; median $=$ 12.85 spikes/s). At all ages, the distribution of SRs was highly skewed, with a mode at rates $<3$ spikes/s (Fig. $3 B$ ). This developmental diversification of SRs and recruitment of high SR ANFs in older animals resemble in vivo data from kittens (Romand, 1984; Walsh and McGee, 1987). However, the maximum firing rates recorded in vitro (55 spikes/s) were lower than those recorded in vivo: up to $\sim 120$ spikes/s for mouse, rat, cat, and rabbit (Kiang et al., 1965; Liberman, 1978; Borg et al., 1988; el Barbary, 1991; Taberner and Liberman, 2005); up to 140 spikes/s for Mongolian gerbils and guinea pig (Evans, 1972; Schmiedt, 1989; Tsuji and Liberman, 1997); and up to 200 
spikes/s for chinchilla (Temchin et al., 2008). The 2 -fold to 3 -fold difference between the maximum SRs recorded in vitro and in vivo could possibly be accounted for by the differences in recording conditions (e.g., room temperature vs body temperature) or by the differences in IHC resting membrane potentials due to the artificial extracellular solution.

To test the effects of temperature on SR, a few fibers (P29-P31) were recorded at elevated temperature (a gradual increase in bath temperature up to $\sim 30^{\circ} \mathrm{C}$ over a few minutes). Most of the fibers showed increased SR during the temperature ramp $(n=10$ of 11). However, only some of the fibers were able to maintain firing at higher SR for at least $5 \mathrm{~min}$ at elevated temperature and then fully recover to their initial SR when the temperature decreased ( $n=2$ of 11; Fig. $3 C$ is one example). The majority of fibers showed a rundown in SR at elevated temperature, or even during the temperature ramp. Selected fibers that showed a stable increase in SR during the temperature ramp were analyzed ( $n=6$ of 11; Fig. 3D). The estimated Q10 from these fibers ranged from 2.38 to 15.71 (median $=6.60)$, and linear rates of increase were $1.93-17.15$ spikes $/\left(\mathrm{s}^{\circ} \mathrm{C}\right)\left(\right.$ median $=6.48$ spikes $\left./\left(\mathrm{s}^{\circ} \mathrm{C}\right)\right)$. The wide range of temperature coefficients observed here overlaps the similarly wide range found in vivo in the caiman (0.2-3.5 spikes $/\left(\mathrm{s}^{\circ} \mathrm{C}\right)$ in Smolders and Klinke (1984), although the values recorded in rat are larger.

\section{IEIs of ANFs recorded in vitro}

The IEI histograms of in vivo ANFs usually show an approximately exponential decay from a peak at the end of the refractory period (Fig. 4E1). Another view of the spiking behavior is obtained from the hazard function (Fig. 4E2), which shows the probability of an event at a particular interval, given that there has been no event so far. The hazard functions of in vivo ANFs are zero during the ARP (e.g., IEIs $<2 \mathrm{~ms}$ in Fig. 4E2), rise smoothly during the next few milliseconds (usually called the relative refractory period), and then are approximately constant or continue to increase slowly at longer intervals (Gray, 1967; Gaumond et al., 1982).

The decay of the IEI histogram from its peak is often not exactly exponential, indicated by the departure of the data from the least-square-fit line in Figure 4E1 (red). Usually, the decay is "faster than exponential", meaning that the histogram bends downward from the line at short and long intervals. This behavior corresponds to the slowly recovering components of refractoriness seen in hazard functions as a gradual rise at longer intervals (Fig. 4E2) (Gaumond et al., 1982). If the decay of the IEI histogram were exactly exponential, then the hazard function would be constant at the horizontal red line, the "hazard rate" equal to the absolute value of the slope of the red line fit to the IEI histogram (as in the example in Fig. 4D3).

In vitro, a subset of extracellularly recorded ANF spike trains showed characteristics similar to in vivo spike trains ( $n=46$ of 83 ; Fig. 4A). The peak-timing of EPSCs also showed similar characteristics ( $n=4$ of 4 ; Fig. $4 B$ ). These include faster than exponential IEI histograms (Grant et al., 2010, their Fig. 5) and a slowly rising hazard function at longer intervals. However, a refractory period at short IEIs was not present in EPSCs and, in some recordings ( $n=3$ of 4 ), there was an excess of short IEIs, as seen by the peak near zero in Figure 4B2, B3 (at $\sim 0.45 \mathrm{~ms}$ when plotted with $0.1 \mathrm{~ms}$ bins). The excess of short intervals was due to the closely spaced peaks within multiphasic EPSCs.

Unexpectedly, a population of ANF spike trains ( $n=37$ of 83) showed preferred IEIs, reflected by a peak at the rising phase of the IEI histograms and hazard functions (Fig. 4C2,C3, green arrows). In some extreme cases, spike trains consisted of AP bursts with highly regular IEIs (Fig. 4D1, inset), producing a large peak in the IEI histogram and hazard function (Fig. $4 D 2, D 3$, green arrows). Such regular IEIs are reminiscent of bursting activity in neonatal ANFs (Tritsch et al., 2010), suggesting that this might be an immature feature still present in postnatal ANFs.

\section{Changes in properties of ANF spike trains after hearing onset}

To study developmental changes of ANF spike trains after hearing onset, several properties were assessed based on hazard function plots. The ARP was measured as the IEI at the 0.1 percentile of the data (to exclude occasional random events), which corresponds well to the duration of the period with minimal spiking at the beginning of the hazard function (Fig. $4 C 3, D 3$, green bars). The preferred IEI was the time of the first peak in the hazard function (Fig. 4C3,D3, green arrows). The prevalence of the preferred IEI was estimated by the relative size of the peak to the steady-state level in the hazard function plot, measured as the hazard rate. Only fibers with a relative peak size $>1.6$ were considered as having a preferred IEI.

Several properties of ANF spike trains changed after hearing onset, mostly during the third week. From P15-P17 to P29-P32, the percentage of fibers with a preferred IEI decreased (Fig. $5 A$ ). The relative peak size (Fig. $5 B$ ) and the duration of the preferred IEI (Fig. 5 C), indicated by the peak position in the hazard function, significantly decreased with age (for statistics, see Fig. $5 C$, legend). These data suggest that the occurrence of preferred IEIs in spike trains is a feature of immature ANFs that gradually diminishes during postnatal weeks 3-5.

After hearing onset, the ARP also significantly shortened (Fig. $5 D$ ). Intriguingly, ANF spike trains with preferred IEIs have significantly longer ARPs than those without (Fig. $5 E$ ), even if data at P15-P17 were excluded to avoid confounding developmental changes. Furthermore, the duration of the ARP was positively correlated with the logarithm of the relative peak size (Fig. 5F). These data suggest that fibers with longer ARPs have a higher likelihood of exhibiting regular firing. Finally, because the preferred IEI usually resides immediately after the ARP in the hazard function plot, the peak position was also positively correlated with the duration of the ARP (Fig. 5G).

The SR of ANFs diversified during the same time period as the aforementioned changes in ANF spike train properties, suggesting possible connections between these two developmental processes. However, ANF spike trains with or without preferred IEIs did not differ in their SR distributions (Fig. $5 H$ ). In addition, there was no significant correlation between SR and the logarithm of the relative peak size (Fig. 5I) or the duration of the ARPs (Fig. 5J).

In summary, several properties of ANF spike trains change during postnatal weeks $3-5$, including a decrease in the contribution of preferred IEIs to spike trains and a shortening of the ARP. Diversification of SR occurs simultaneously with these changes, but most likely through independent mechanisms.

\section{Presynaptic and postsynaptic activation of spontaneous activity in immature ANFs}

To investigate the origin of the preferred IEIs in the ANF spike trains and to test whether there might be endogenous firing in the postsynaptic ANFs, synaptic transmission was eliminated by either blocking calcium channels with cadmium chloride or by blocking glutamate receptors (GluRs). 
A1

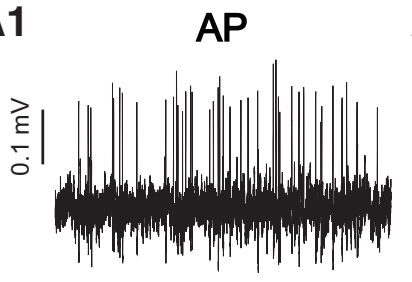

B1

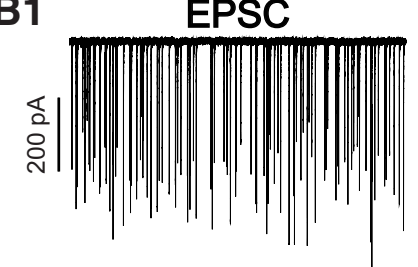

C1

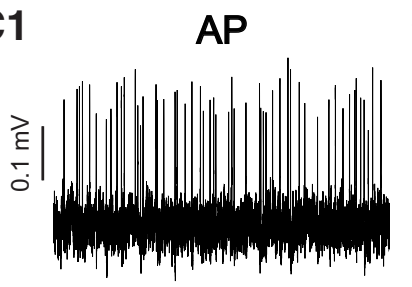

D1

AP
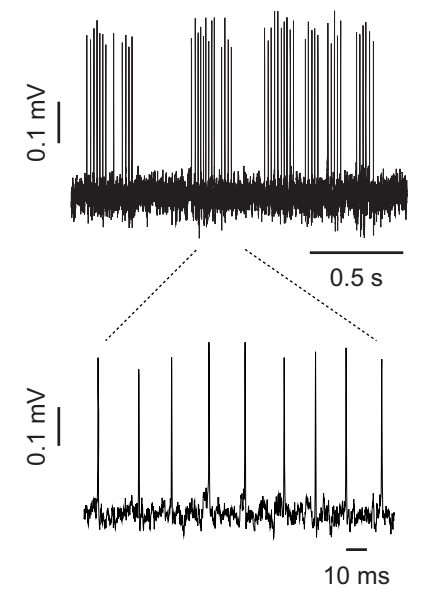

A2

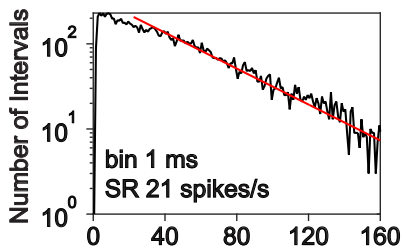

B2

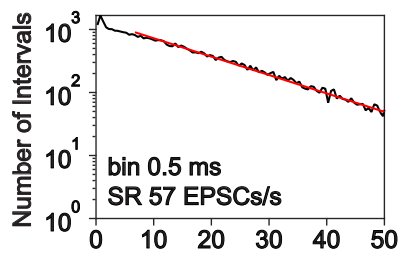

C2

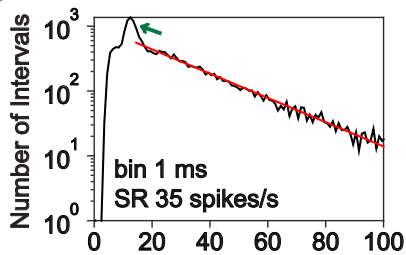

D2

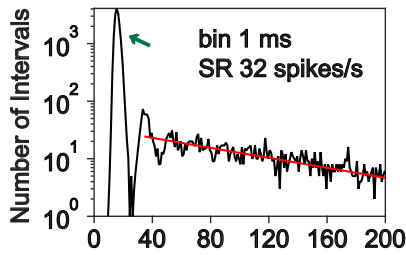

E1

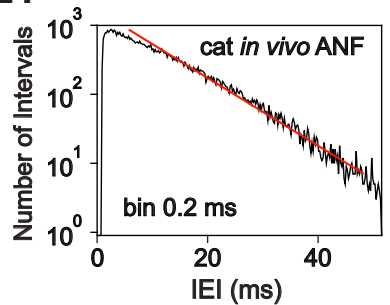

A3

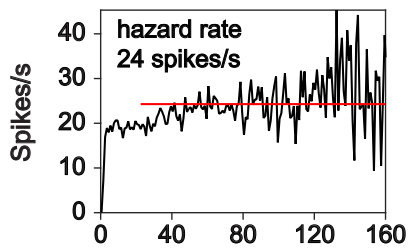

B3

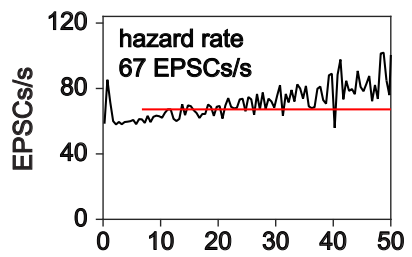

C3

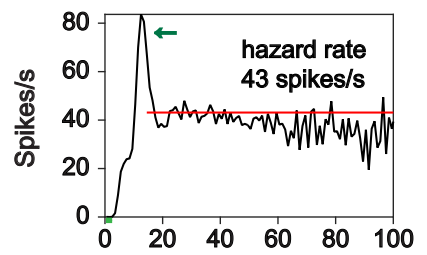

D3

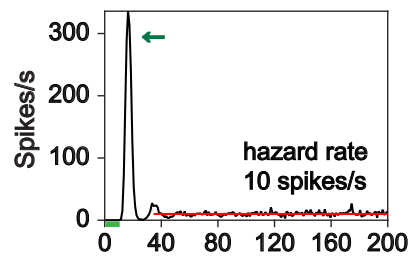

E2

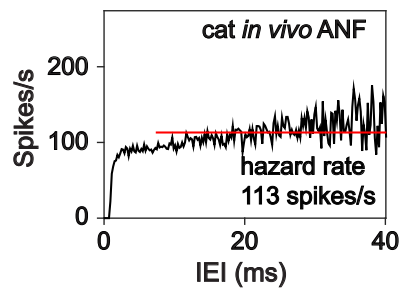

Figure 4. Characteristics of in vitro ANF recordings. $A-D$, Example traces $(A 1, B 1, C 1, D 1)$, El histogram $(A 2, B 2, C 2, D 2)$, and hazard function plots $(A 3, B 3, C 3, D 3)$ of in vitro ANF recordings in rat. $A, C, D$, Spikes in loose-patch extracellular recordings. $B$, EPSCs in an intracellular recording. Short intervals with a few events in the hazard function represent the ARP (e.g., $\mathbf{3}_{2}, \mathbf{D} 3$, green bars). Some recordings exhibited a peak at IEIs slightly larger than the ARP (green arrows), demonstrating the preferred occurrence of these IEls. Bin size for IEI histogram and hazard function plots are shown on the IEl histogram. D1, Inset, One of the AP bursts in D1 at an expanded time scale, demonstrating the regular intervals between APs; these form the large peak at the green arrows in D2, D3. E, IEI histogram and hazard function plot of an in vivo ANF recording in cat. Red lines indicate least-squares fits to the IEI histogram; they show exponential decay of IEl probability for reference. The hazard rate is the absolute value of the slope of these lines; the hazard rate is shown as a horizontal red line on the hazard functions. Note slightly faster than exponential decay in most examples $(A 2, B 2, E 1)$.

In cadmium chloride $(0.5 \mathrm{~mm})$, spontaneous activity was completely blocked in fibers without preferred IEIs (median $=$ $100 \% ; n=14$ of 14; P15-P31; Fig. 6 A, navy blue circles), but only partially blocked in the majority of fibers with preferred IEIs (median $=70 \%$; partial block in 6 of 8 fibers, ranging from $0 \%$ to $87 \%$; Fig. $6 A$, blue triangles). These results suggest that, in spike trains with preferred IEIs, some of the APs were initiated intrinsically in the fiber rather than by synaptic transmission.

In GluR blockers (either NBQX, or a mixture to block all subgroups of GluRs; see Fig. 6A, legend), spontaneous activity was only partially blocked in most fibers with preferred IEIs (median $=30 \% ; n=14$ of 15,0\%-97\% block; Fig. 6 B, blue triangles), consistent with the data in cadmium chloride. However, in fibers without preferred IEIs, where cadmium chloride had caused a complete block, GluR blockers produced only partial block in more than half of the fibers (median $=90 \% ; n=8$ of $14,0 \%-$ $96 \%$ block; Fig. $6 B$, navy blue circles). These data suggest that, in both groups of fibers, with and without preferred IEI, some of the APs were initiated intrinsically in the fiber. The complete block by cadmium in fibers without preferred IEI could reflect the additional involvement of postsynaptic calcium channels in a population of intrinsically generated APs.

Consistent with the decline of intrinsic firing in ANFs during posthearing maturation, the average percentage of SR reduction by GluR blockers increased from $41 \%$ at P15-P18 $(n=9)$, to $46 \%$ at P19-P21 $(n=11)$ and to $75 \%$ at P29-P32 $(n=10)$. 
A

E

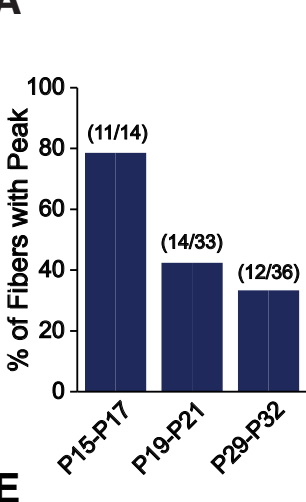

P19-P21, P29-P32
B

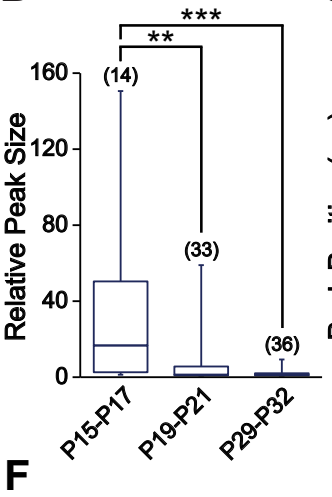

C

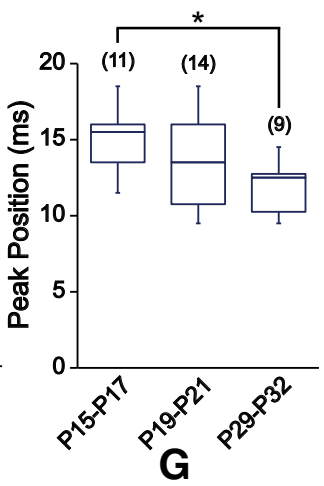

D

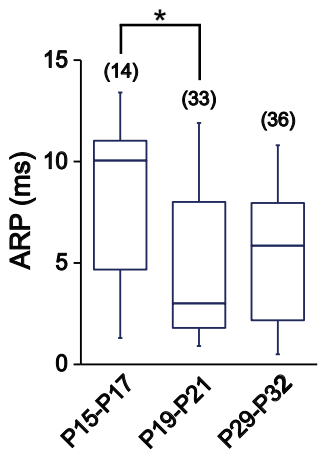

With Peak

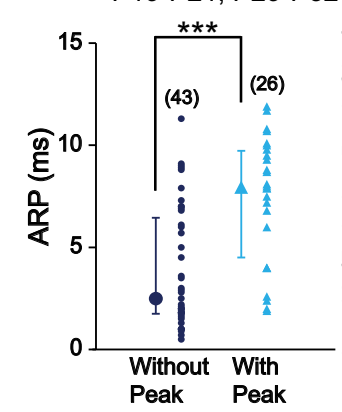

H P19-P21, P29-P32

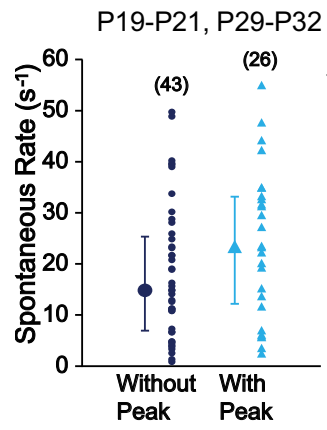

ALL
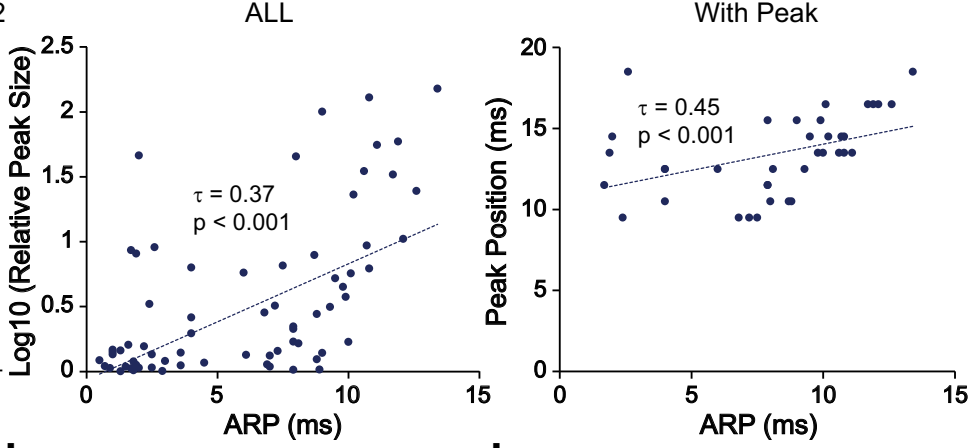

J
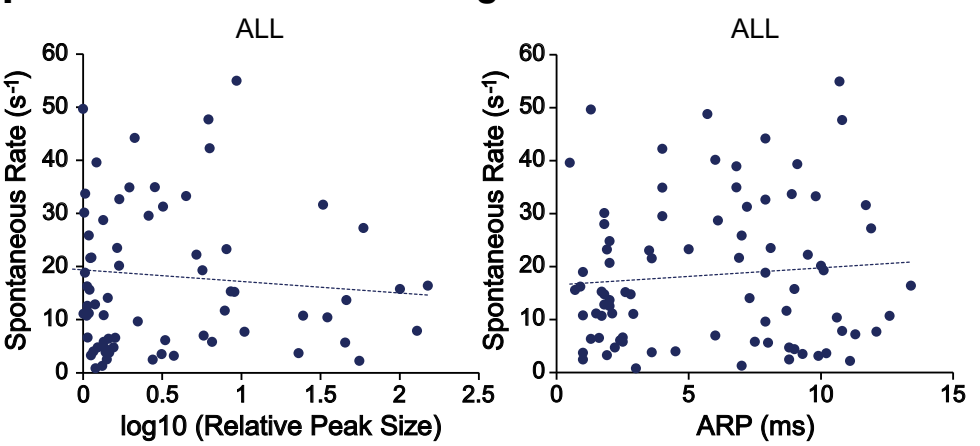

Figure 5. Changes in the properties of ANF spike trains with age. $A-D$, Histogram and box plots represent properties of ANF spike trains in three age groups. $A$, The percentage of fibers with peak in their hazard function plot decreased from $79 \%$ at P15-P17 to $42 \%$ at P19-P21 and then to $33 \%$ at P29-P32. B, The relative peak size decreased significantly over age $\left(H_{(2)}=16.91, p=0.0002\right)$ : from P15-P17 (median $\left.=16.75\right)$ to P19-P21 (median $\left.=1.35, p=0.003\right)$ and to P29-P32 (median $\left.=1.23, p=0.78\right)($ between P15-P17 and P29-P32, $p=$ $0.0003)$. C, The peak time for fibers with peak shifted to significantly smaller values over age $\left(H_{(2)}=8.77, p=0.01\right)$ : from P15-P17 (median $\left.=15.5 \mathrm{~ms}\right)$ to P19-P21 (median $=13.5$ $\mathrm{ms}, p=0.35$ ), and to P29-P32 (median $=12.5 \mathrm{~ms}, p=0.28$ ) (between P15-P17 and P29-P32, $p=0.01$ ). $\boldsymbol{D}$, The ARP was significantly shortened over age $\left(H_{(2)}=7.78, p=0.02\right):$ from $\mathrm{P} 15-\mathrm{P} 17$ (median $=10.05 \mathrm{~ms}$ ) to P19-P21 (median $=3 \mathrm{~ms}, \boldsymbol{p}=0.03$ ) and to P29-P32 (median $=5.85 \mathrm{~ms}, \boldsymbol{p}=0.88$ ) (between P15-P17 and P29-P32, $p=0.06$ ). $\boldsymbol{B}-\boldsymbol{D}, \mathrm{K}$ Kuskal - Wallis rank sum test was used with post hoc pairwise comparisons using Nemenyi test with $\chi^{2}$ approximation for independent samples. $\chi^{2}$ values were corrected for ties present in the data. $E$, Among recordings obtained at P19-P21 and P29-P32, the ARP of fibers with peak (blue triangle, median $=7.95 \mathrm{~ms}$ ) was significantly longer than those without peak (navy blue dot, median $\left.=2.5 \mathrm{~ms}, W=900.5, p=2.40 \times 10^{-5}\right) . F$, Among all fibers, the duration of the ARP was significantly correlated with the logarithm of the relative peak size $(\tau=0.37, p=$ $\left.7.60 \times 10^{-7}\right) . \mathbf{G}$, Among all fibers with a peak, the duration of the ARP was significantly correlated with peak position $(\tau=0.45, p=0.0002)$. $\boldsymbol{H}$, Fibers with peak (blue triangle, median $=23.39$ spikes/s) did not differ significantly in their median SRs from fibers without peak (navy blue dot, median $=14.82$ spikes $/ \mathrm{s}, \boldsymbol{W}=696, p=0.09$ ). $\boldsymbol{E}, \boldsymbol{H}$, The median of group data with first and third quartiles (error bars) is shown left to the individual data. Wilcoxon rank sum test was used due to non-normal data distributions or the small sample size. $I, J$, Among all fibers, SR did not have a significant correlation with either the logarithm of the relative peak size $(\tau=-0.10, p=0.17)$ or the duration of the ARP $(\tau=0.02, p=0.74)$. Only fibers with spike counts $\geq 2000$ were included in this analysis. ${ }^{*} p<0.05 .{ }^{* *} p<0.01$. ${ }^{* * *} p<0.001$.

Additional support for endogenous spiking was obtained from AP waveform analysis in two fibers that had remaining activity in NBQX. When APs were examined for the prepotential that corresponds to the EPSP, a subset of APs showed inflections in the rising phase before NBQX (Fig. 6C, Before NBQX left and middle, the APs in blue). The average AP waveform of this recording also showed an inflection in the rising phase, reflected as a local minimum in the first derivative (Fig. $6 \mathrm{C}$, Before NBQX right). These inflections in the rising phase of APs were lost during NBQX application (Fig. 6C, During NBQX), suggesting that the remaining APs (red) were not activated by EPSPs, but generated intrinsically by the ANF.

$\mathrm{AP}$ waveforms were also analyzed in two additional fibers that contained AP bursts with preferred IEIs (Fig. 6D, left). In a subset of those bursts, the initial AP showed an inflection in the rising phase (Fig. $6 D$, burst a), whereas the following APs did not, suggesting that the initial AP was initiated by an EPSP, whereas the following APs were most likely initiated intrinsically in the fiber. In the majority of the remaining bursts (Fig. $6 D$, burst b), none of the APs showed an inflection in the rising phase, suggesting that 
A

$$
\mathrm{Cd}^{2+} \text { Block }
$$

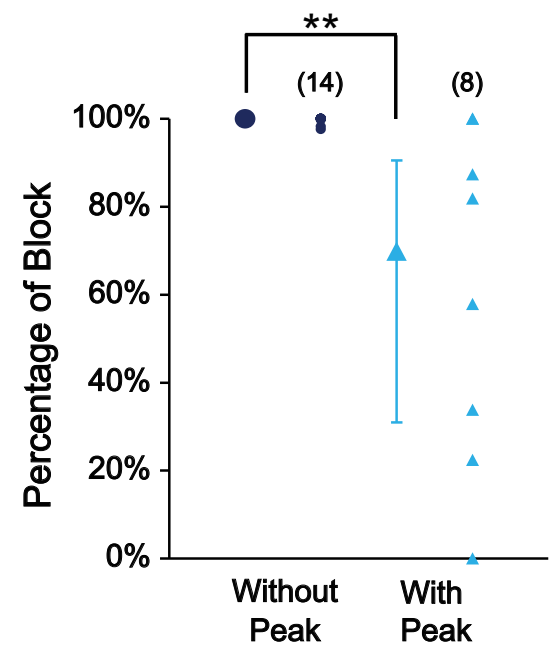

C
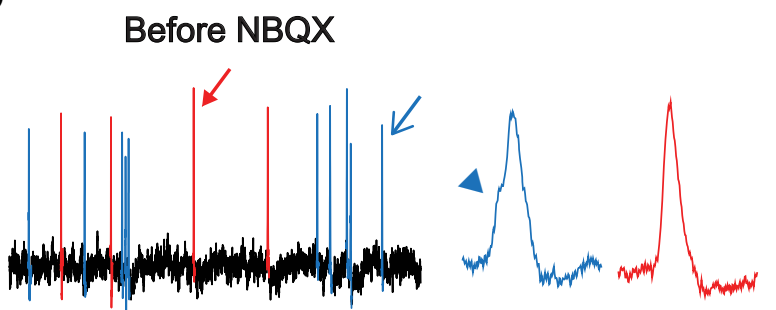

छे।

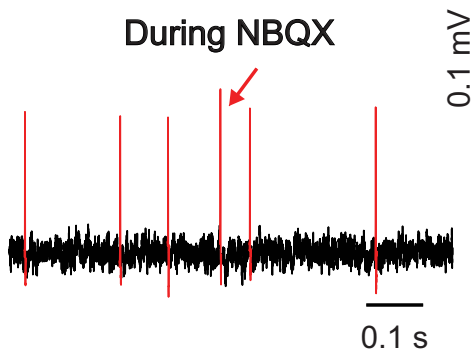

D

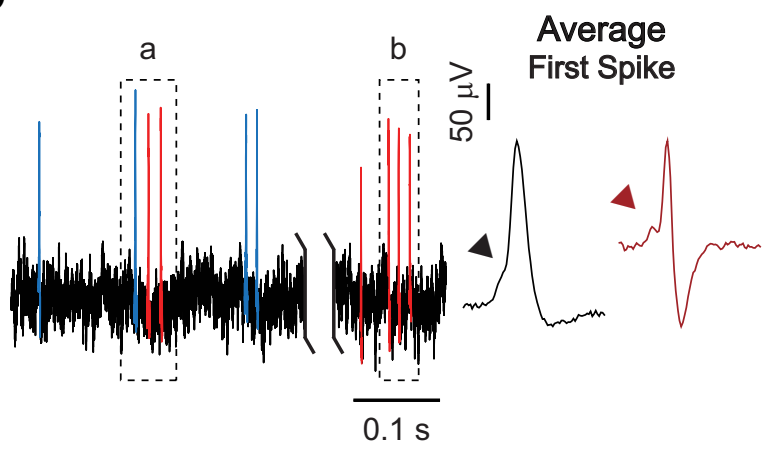

\section{GluR Block}

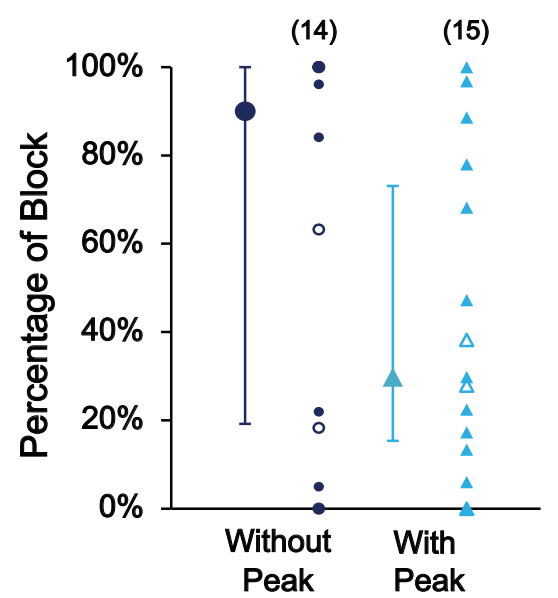

all of them were initiated intrinsically. Although inflections in the rising phase were occasionally observed in the following spikes in a burst, the average waveform of the following spikes did not show such a feature, compared with the average waveform of the initial spikes (Fig. $6 D$, middle, right).

In summary, pharmacological tests and waveform analysis of ANF spike trains suggest that APs are either activated by IHC synaptic transmission or by intrinsic postsynaptic mechanisms in the ANF. Intrinsic firing gradually decreases during posthearing maturation.

\section{Comparison of SRs and spike timing between two fibers contacting the same IHC}

With in vivo single-unit recordings and backfilling of ANFs, it has been shown in cat and guinea pig that low SR fibers have smaller calibers and contact IHCs preferentially on the modiolar side, whereas high SR fibers have larger calibers and make preferential contacts on the pillar side (Liberman, 1982; Tsuji and Liberman, 1997). Second, EM studies have shown that individual IHCs are contacted by both smaller-caliber fibers on the modiolar side and larger-caliber fibers on the pillar side (Liberman, 1980). From these combined observations, it has been concluded that individual IHCs are contacted by ANFs with different SRs.

To directly test this hypothesis, simultaneous recordings from two ANF endings contacting the same IHC were performed. The age range for recordings was P19-P32, as the diversification of SRs had already occurred at this age (Fig. 3). Sixteen such paired recordings were performed, and the range of SRs for the 32 fibers in this dataset $(0-42.15$ spikes/s;

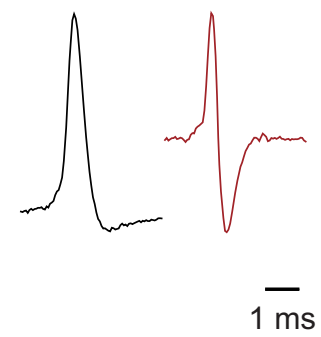

Figure 6. Presynaptic and postsynaptic origin of spontaneous activity in ANFs. A, Percentage of spontaneous activity blocked by addition of $0.5 \mathrm{~mm} \mathrm{CdCl}$ to block calcium channels. The significant higher percentage of block that was achieved in fibers without peak (without preferred IEls, navy blue circles, median $=100 \%$ ) compared with fibers with peak (with preferred IEls, blue triangles, median $=70 \%, W=17, p=0.003$ ) suggests the presence of nonsynaptically activated APs in fibers with preferred IEls. $\boldsymbol{B}$, Percentage of spontaneous activity blocked by NBQX (filled symbols; $10-50 \mu \mathrm{m}$ ) or GluR blocker mixture (open symbols; $20 \mu \mathrm{m}$ NBQX, $20 \mu \mathrm{m}(R S)$-CPP for NMDA receptors, $1 \mathrm{~mm}(R S)$ - $\alpha$-methyl-4-carboxyphenylglycine for mGluRs, $50 \mu \mathrm{m}$ CNQX for kainate/ AMPA receptors). Percentage of block was not significantly different between fibers with (open and filled blue triangles, median = $30 \%$ ) and without preferred IEls (open and filled navy blue circles, median $=90 \%, W=74, p=0.18$ ). $\boldsymbol{A}, \boldsymbol{B}$, The median of group data with first and third quartiles (error bars) is shown left to the individual data. $\boldsymbol{A}, \boldsymbol{B}$, The percentage of block was calculated by comparing the average rates of $1 \mathrm{~min}$ recording segments before and after $2 \mathrm{~min}$ of drug application. Wilcoxon rank sum test was used due to non-normal data distributions. C, D, APs were color-coded according to the waveform. Blue represents APs with inflections (arrowhead) in the rising phase. Red represents APs without inflections in the rising phase. $\boldsymbol{C}$, Left,

Fig. $7 A$ ) was representative of the SR range

Representative traces of one fiber before or during NBQX application. Middle, Events indicated by arrows in traces on the left are enlarged for better visualization of the detailed waveform. Right, Average waveform and its first derivative for events in this recording before or during NBQX application. Arrowheads point to inflections in the rising phase. Events with inflections in the rising phase were lost during NBQX application, suggesting that they were initiated by synaptic activity. $\boldsymbol{D}$, Left, Representative trace of one fiber with preferred IEls, in which two bursts are identified as "a" and "b." The first AP in burst "a" shows an inflection in the rising phase but not the following APs. None of the APs in burst "b" shows an inflection in the rising phase. Middle, Right, Average waveform and its first derivative for the first spikes and the following spikes, respectively, within 50 identified bursts in this recording. ${ }^{* *} p<0.01$. 
A

\begin{tabular}{|c|c|c|}
\hline \multirow{2}{*}{ Pair No. } & \multicolumn{2}{|c|}{ Spon. Rate $\mathbf{s}^{-1}$ ) } \\
\cline { 2 - 3 } & Fiber 1 & Fiber 2 \\
\hline$\# 1$ & 2.01 & 0.13 \\
\hline$\# 2$ & 1.91 & 2.39 \\
\hline$\# 3$ & 18.30 & 0.01 \\
\hline$\# 4$ & 0.31 & 8.01 \\
\hline$\# 5$ & 21.67 & 0.10 \\
\hline$\# 6$ & 0.63 & 18.25 \\
\hline$\# 7$ & 1.53 & 4.63 \\
\hline$\# 8$ & 11.74 & 41.04 \\
\hline$\# 9$ & 42.15 & 16.29 \\
\hline$\# 10$ & 17.30 & 4.02 \\
\hline$\# 11$ & 23.32 & 11.57 \\
\hline$\# 12$ & 39.71 & 27.81 \\
\hline$\# 13$ & 23.09 & 25.87 \\
\hline$\# 14$ & 24.62 & 19.03 \\
\hline$\# 15$ & 4.45 & 10.96 \\
\hline$\# 16$ & 40.78 & 6.50 \\
\hline \multicolumn{3}{|c|}{ Undefined } \\
& Without peak \\
& With peak \\
\hline
\end{tabular}

B1

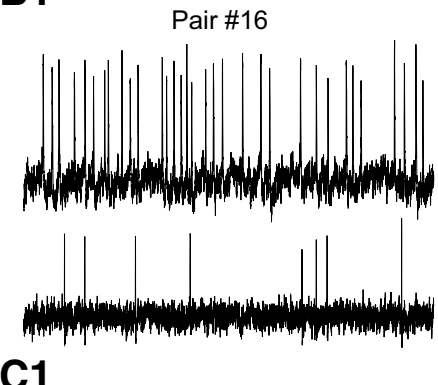

Pair \#14

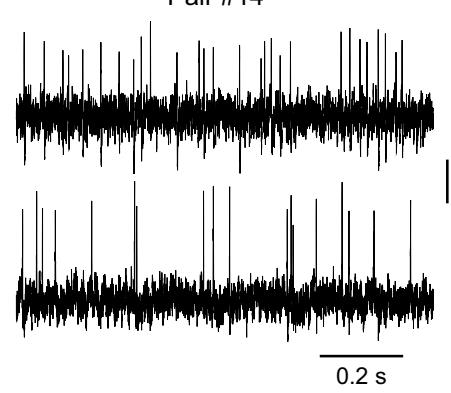

B2
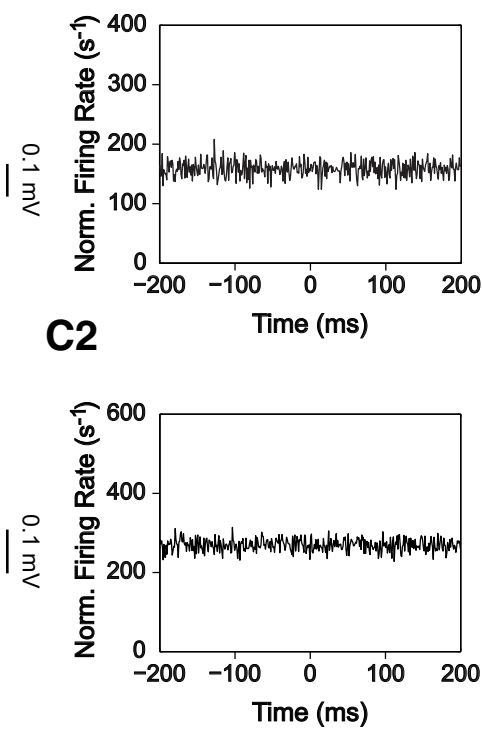

Figure 7. Double recordings of two ANFs contacting the same IHC. $\boldsymbol{A}$, For 16 pairs, two ANFs (fiber 1 and 2 ) that terminate on the same IHC were recorded simultaneously and showed different SRs. B1, C1, Representative traces from two paired recordings of ANFs contacting the same IHC. B2, C2, Cross-correlograms of spike timing for two ANF pairs in B1 and C1, respectively. Bin size is $1 \mathrm{~ms}$.

in the whole population (Fig. 3; $p=0.89$, Kolmogorov-Smirnov test comparing the data in Fig. 7A with the data in Fig. 3; P19-P21, P29-P32 groups, excluding fibers included in Fig. $7 A$ ). We found that two fibers contacting the same IHC could have either similar SRs, such as pair 13, with SRs of 23.09 spikes/s and 25.87 spikes/s, or quite different SRs, such as pair 3, with SRs of 18.30 spikes/s and 0.01 spikes/s or pair 16, with SRs of 40.78 spikes/s and 6.50 spikes/s (Fig. 7B1). There was no correlation of the SRs between two fibers in a pair (Kendall's $\tau=0.15, p=0.45$ ). Furthermore, the distribution of ratios of SRs for two fibers in a pair was not statistically different from that obtained by random choice from the total distribution of the SRs in Figure 3 (P19-P21, P29-P32 groups; $\mathrm{p} \approx 0.80$, Kolmogorov-Smirnov test).

The 10-30 ANF endings contacting a single IHC are thought to function as independent parallel channels, with the stochastic openings of the presynaptic calcium channels localized at individual ribbon synapses most likely setting the spike timing for individual ANFs (Brandt et al., 2005; Moser et al., 2006). To test whether spontaneous activity in ANFs contacting the same IHC behaves independently, spike timing was compared between the two simultaneously recorded fibers. For correlation analysis, a selected window of the paired recording was chosen that lasted for $\geq 7 \mathrm{~min}$, starting from $2 \mathrm{~min}$ after having acquired both fiber recordings. Any correlation between the two fibers would manifest as a peak in the cross-correlogram. No correlation was observed for 16 paired recordings (e.g., Fig. 7B2,C2), suggesting that individual fibers contacting the same IHC spike independently at rest.

A caveat for interpreting the data presented here is that some fibers still showed some immature features in their spike trains and exhibited preferred IEIs ("with peak" in the hazard function, 5 fibers marked in dark green, Fig. 7A), and, as mentioned above, these APs may be initiated postsynaptically. However, when only fiber pairs without preferred IEIs in their spike trains were compared ("without peak", fiber pairs 13-16, Fig. 7A), the same observations were made: (1) two fibers contacting the same IHC can have different SRs; and (2) their spike timing did not show any correlation. These data suggest that indeed the basic mechanisms underlying the spike timing of individual ANFs reside at the individual IHC/ANF synapses and that those synapses act independently at rest.

\section{Discussion}

This study characterized the development of spontaneous activity in ANFs with loose-patch extracellular recordings using in vitro rat cochlear preparations. The results demonstrate posthearing-onset diversification of SRs and changes in the properties of ANF spike trains in the first postnatal month. In older preparations, the statistical properties of spike trains, including IEIs, refractoriness, and spike counting statistics, match those observed in vivo in adult animals. With the exception of refractoriness, these properties are also observed in intracellularly recorded EPSCs at the afferent terminals. These results show that in vitro recordings from ANFs in 3- to 4-week-old rats provide a valid model for investigating the underlying mechanisms that account for different SRs and stimulus response patterns in ANFs. Paired recordings of ANFs contacting the same IHC show that fibers with different SRs contact the IHC but discharge independently, with no correlation in their spike timing.

\section{Sources of ANF firing properties}

Our analyses show that several temporal properties of ANF spike trains in young adult rats (P19-P32) derive from properties of the presynaptic release process. These include the fractal behavior captured by the Fano factor analysis (Fig. 2) and several properties revealed by IEI or hazard function analysis (Fig. 4); the exception is refractoriness, which is absent in EPSC recordings. Because EPSC timing directly reflects the properties of the presynaptic release process, these results clearly assign the properties of spike trains studied here to the presynaptic release mechanism, as opposed to an intrinsic mechanism of AP initiation in the afferent terminals. Refractoriness, of course, is a property of the 
AP generation mechanisms in the postsynaptic terminal. This conclusion supports the idea, first suggested as a model of ANF synaptic function (Gaumond et al., 1982; Young and Barta, 1986; Johnson, 1996; Kelly et al., 1996; Meddis and O’Mard, 2005; Heil et al., 2007; Peterson et al., 2014), that the spike trains of ANFs represent a random release of packets of neurotransmitter from the hair cell, which induce a postsynaptic spike when the postsynaptic effect exceeds threshold and the fiber is not refractory.

\section{Diversification of SRs after hearing onset, in vivo and in vitro} Development of ANF spontaneous activity has been described in kitten, mouse, and Mongolian gerbil (Romand, 1984; Walsh and McGee, 1987; Müller, 1996; Wong et al., 2013) with features similar to those presented here in rat: only low SR fibers were observed perihearing onset, with a gradual recruitment of high SR fibers after hearing onset. The distribution of rat ANF SRs at the oldest ages (Fig. 3B; P29-P32 group) is consistent with studies in rat and mouse (el Barbary, 1991; Taberner and Liberman, 2005), despite the narrower range of overall SRs observed at room temperature in vitro. When recording temperature was raised, SR increased by an amount that varied from fiber to fiber (Fig. 3D). At $30^{\circ} \mathrm{C}$, which is still below in vivo body temperature, the highest SRs in our data are similar to the highest SRs observed in vivo. This result suggests that the range of SRs in Figure $3 A$ would expand to cover the in vivo range if experiments could be done at body temperature.

Recordings in this study were obtained from the cochlear apical turn, a region representing $1-5 \mathrm{kHz}$ sound frequencies (Müller, 1991). Previous studies have shown a relatively homogeneous distribution of SRs across different characteristic frequencies in rat, mouse, cat, and guinea pig (Kiang et al., 1965; Liberman, 1978; el Barbary, 1991; Tsuji and Liberman, 1997; Taberner and Liberman, 2005), and to a lesser extent in Mongolian gerbil and chinchilla (Schmiedt, 1989; Temchin et al., 2008; Bourien et al., 2014). Thus, the limited sampling region in this study should not prevent a complete representation of the range of SRs.

\section{Maturation of ANF spike train properties after hearing onset} The existence of preferred IEIs in posthearing ANF spike trains is reflected in peaks in the IEI and hazard functions (Fig. 4C,D). The phenomenon shown in vitro here is reminiscent of preferred IEIs shown by some ANFs in vivo in adult animals, namely, a very brief peak ( $<1 \mathrm{~ms})$ right at the end of the ARP in the hazard function (Gaumond et al., 1982; Miller and Mark, 1992). This phenomenon is predicted by Hodgkin-Huxley style models of the IHC/ ANF synapse in which the rapidly dropping threshold at the end of the ARP can be crossed by residual excitation of the postsynaptic terminal (Miller and Wang, 1993). These data beg the question of whether the preferred IEIs in the in vivo data are similar to the preferred IEIs shown here. However, there are substantial quantitative differences between the two phenomena, including duration, amplitude, and latency of the peaks. Moreover, the peaks in the in vivo data have been attributed to technical problems of event detection at short IEIs (Gaumond et al., 1982; Heil et al., 2007).

The prevalence of preferred IEIs in ANF spike trains declines with age (Fig. 5), consistent with an intrinsic postsynaptic spiking mechanism remaining from prehearing-onset animals (Tritsch et al., 2010) that disappears as the fibers mature. Intrinsic firing in immature ANFs may play an important role in auditory circuit maturation before hearing onset. In support of this idea, cochlear ablation in gerbils and mice before hearing onset causes degeneration of cochlear nucleus neurons (Hashisaki and Rubel, 1989;
Tierney et al., 1997; Mostafapour et al., 2000). In contrast, formation and organization of brainstem auditory pathways seem unaffected in the congenital deafness $d n / d n$ mouse (Youssoufian et al., 2005, 2008; for review, see Wang and Bergles, 2015). Assuming that the $d n / d n$ mutation affects only hair cells, intrinsic spontaneous firing of ANFs in $d n / d n$ mice could still contribute to the establishment of the auditory circuit, despite the loss of hair-cell function. Consistent with this hypothesis, cochlear ablation after hearing onset does not affect the survival of cochlear nucleus neurons (Hashisaki and Rubel, 1989; Tierney et al., 1997; Mostafapour et al., 2000). Our results are consistent with these data by showing an intrinsic firing mode before and shortly after hearing onset, which could be important in organizing central synaptic function. Following hearing onset, this is gradually replaced by an independent random-firing encoding mode optimized to represent the information from IHCs across acoustic environments.

\section{Functional implications of uncorrelated spike timing in ANFs contacting the same IHC}

Previous studies have reported independent firing of ANFs with the same best frequencies in vivo (Johnson and Kiang, 1976; Young and Sachs, 2008); here we tighten that finding by showing that spike times during spontaneous activity of ANFs contacting the same IHC are uncorrelated (Fig. 7).

The lack of correlation is important from the perspective of how information about the degree of activation of a hair cell is communicated to the brain. There are $\sim 10-30$ ANFs contacting an IHC (Liberman et al., 1990; Stamataki et al., 2006). Given that the encoding of sounds by one IHC/ANF synapse is noisy, information transfer is maximized by multiple independent sources of information about the activation of the hair cell, meaning independent neurons (Winslow and Sachs, 1988; Cover and Thomas, 1991; Heinz et al., 2001). In addition, low and high SR ANFs have different thresholds for activation by sound and different dynamic ranges, so that high SR fibers respond to low-level stimuli and low SR fibers maintain their dynamic sensitivity to sounds at moderate-to-high sound levels where high SR fibers are saturated (Sachs and Abbas, 1974; Geisler et al., 1985; Yates, 1990). Thus, multiple fibers provide a representation of the full dynamic range of the hair cell, retaining good sensitivity to changes in IHC activation over the entire range (Winslow and Sachs, 1988).

Another implication of the apparent independence of synapses on a hair cell is that, in the presence of a stimulus, there will be stimulus-driven correlation among the neurons connected to one IHC, but no "noise correlation" (Averbeck et al., 2006), maximizing information transfer. Mechanistically, independence is consistent with vesicle exocytosis being triggered by separate calcium nanodomains (Brandt et al., 2005; Goutman and Glowatzki, 2007). When IHCs become sufficiently depolarized, one could hypothesize that large calcium influx may cause a merge of calcium domains and correlated transmitter release between nearby ribbons. If caused by stimulation, this merging might produce additional stimulus correlation. However, it might also produce noise correlation (e.g., if the variability in one domain dominates calcium in nearby synapses). Stimulus correlation has been observed between pairs of ANFs, for stimuli with envelopes, like a low-frequency tone or a broadband noise; but noise correlations have not been observed (Johnson and Kiang, 1976; Young and Sachs, 2008), speaking against the idea of merging calcium domains. 


\section{References}

Averbeck BB, Latham PE, Pouget A (2006) Neural correlations, population coding, and computation. Nat Rev Neurosci 7:358-366. CrossRef Medline

Blatchley BJ, Cooper WA, Coleman JR (1987) Development of auditory brainstem response to tone pip stimuli in the rat. Brain Res 429:75-84. Medline

Borg E, Engström B, Linde G, Marklund K (1988) Eighth nerve fiber firing features in normal-hearing rabbits. Hear Res 36:191-201. CrossRef Medline

Bourien J, Tang Y, Batrel C, Huet A, Lenoir M, Ladrech S, Desmadryl G, Nouvian R, Puel JL, Wang J (2014) Contribution of auditory nerve fibers to compound action potential of the auditory nerve. J Neurophysiol 112:1025-1039. CrossRef Medline

Brandt A, Khimich D, Moser T (2005) Few CaV1.3 channels regulate the exocytosis of a synaptic vesicle at the hair cell ribbon synapse. J Neurosci 25:11577-11585. CrossRef Medline

Brooks CM, Eccles JC (1947) Electrical investigation of the monosynaptic pathway through the spinal cord. J Neurophysiol 10:251-273. Medline

Buzsáki G, Penttonen M, Nadasdy Z, Bragin A (1996) Pattern and inhibition-dependent invasion of pyramidal cell dendrites by fast spikes in the hippocampus in vivo. Proc Natl Acad Sci U S A 93:9921-9925. CrossRef Medline

Cover TM, Thomas JA (1991) Elements of information theory. New York: Wiley-Interscience.

Cox DR, Lewis P (1978) The statistical analysis of a series of events. London: Metheun.

Ehret G (1983) Development of hearing and response behavior to sound stimuli: behavioral studies. In: Development of auditory and vestibular systems (Romand R, ed), pp 211-237. San Diego: Academic.

el Barbary A (1991) Auditory nerve of the normal and jaundiced rat: I. Spontaneous discharge rate and cochlear nerve histology. Hear Res 54: 75-90. CrossRef Medline

Evans EF (1972) The frequency response and other properties of single fibres in the guinea-pig cochlear nerve. J Physiol 226:263-287. CrossRef Medline

Fatt P (1957) Electric potentials occurring around a neurone during its antidromic activation. J Neurophysiol 20:27-60. Medline

Fay MP, Shaw PA (2010) Exact and asymptotic weighted logrank tests for interval censored data: the interval $\mathrm{R}$ package. J Stat Softw 36:1-34. Medline

Freygang WH Jr, Frank K (1959) Extracellular potentials from single spinal motoneurons. J Gen Physiol 42:749-760. CrossRef Medline

Furman AC, Kujawa SG, Liberman MC (2013) Noise-induced cochlear neuropathy is selective for fibers with low spontaneous rates. J Neurophysiol 110:577-586. CrossRef Medline

Gaumond RP, Molnar CE, Kim DO (1982) Stimulus and recovery dependence of cat cochlear nerve fiber spike discharge probability. J Neurophysiol 48:856-873. Medline

Geal-Dor M, Freeman S, Li G, Sohmer H (1993) Development of hearing in neonatal rats: air and bone conducted ABR thresholds. Hear Res 69:236242. CrossRef Medline

Geisler CD, Deng L, Greenberg SR (1985) Thresholds for primary auditory fibers using statistically defined criteria. J Acoust Soc Am 77:1102-1109. CrossRef Medline

Glowatzki E, Fuchs PA (2002) Transmitter release at the hair cell ribbon synapse. Nat Neurosci 5:147-154. CrossRef Medline

Goutman JD, Glowatzki E (2007) Time course and calcium dependence of transmitter release at a single ribbon synapse. Proc Natl Acad Sci U S A 104:16341-16346. CrossRef Medline

Grant L, Yi E, Glowatzki E (2010) Two modes of release shape the postsynaptic response at the inner hair cell ribbon synapse. J Neurosci 30:42104220. CrossRef Medline

Gray PR (1967) Conditional probability analyses of the spike activity of single neurons. Biophys J 7:759-777. CrossRef Medline

Hashisaki GT, Rubel EW (1989) Effects of unilateral cochlea removal on anteroventral cochlear nucleus neurons in developing gerbils. J Comp Neurol 283:5-73. Medline

Heil P, Neubauer H, Irvine DR, Brown M (2007) Spontaneous activity of auditory-nerve fibers: insights into stochastic processes at ribbon synapses. J Neurosci 27:8457-8474. CrossRef Medline

Heinz MG, Young ED (2004) Response growth with sound level in auditory-nerve fibers after noise-induced hearing loss. J Neurophysiol 91:784-795. CrossRef Medline

Heinz MG, Colburn HS, Carney LH (2001) Rate and timing cues associated with the cochlear amplifier: level discrimination based on monaural cross-frequency coincidence detection. J Acoust Soc Am 110:2065-2084. CrossRef Medline

Henze DA, Borhegyi Z, Csicsvari J, Mamiya A, Harris KD, Buzsáki G (2000) Intracellular features predicted by extracellular recordings in the hippocampus in vivo. J Neurophysiol 84:390-400. Medline

Hothorn T, Hornik K, van de Wiel MA, Zeileis A (2008) Implementing a class of permutation tests: the coin package. J Stat Softw 28:1-23.

Jewett DL, Romano MN (1972) Neonatal development of auditory system potentials averaged from the scalp of rat and cat. Brain Res 36:101-115. CrossRef Medline

Johnson DH (1996) Point process models of single-neuron discharges. J Comput Neurosci 3:275-299. CrossRef Medline

Johnson DH, Kiang NY (1976) Analysis of discharges recorded simultaneously from pairs of auditory nerve fibers. Biophys J 16:719-734. CrossRef Medline

Kelly OE, Johnson DH, Delgutte B, Cariani P (1996) Fractal noise strength in auditory-nerve fiber recordings. J Acoust Soc Am 99:2210-2220. CrossRef Medline

Kiang NY, Watanabe T, Thomas EC, Clark LF (1965) Spontaneous activity. In: Discharge patterns of single fibers in the cat's auditory nerve. Cambridge, MA: Massachusetts Institute of Technology.

Kumar AR, Johnson DH (1993) Analyzing and modeling fractal intensity point processes. J Acoust Soc Am 93:3353-3373. CrossRef Medline

Li GL, Keen E, Andor-Ardó D, Hudspeth AJ, von Gersdorff H (2009) The unitary event underlying multiquantal EPSCs at a hair cell's ribbon synapse. J Neurosci 29:7558-7568. CrossRef Medline

Li J, Young ED (1993) Discharge-rate dependence of refractory behavior of cat auditory-nerve fibers. Hear Res 69:151-162. CrossRef Medline

Liberman MC (1978) Auditory-nerve response from cats raised in a lownoise chamber. J Acoust Soc Am 63:442-455. CrossRef Medline

Liberman MC (1980) Morphological differences among radial afferent fibers in the cat cochlea: an electron-microscopic study of serial sections. Hear Res 3:45-63. CrossRef Medline

Liberman MC (1982) Single-neuron labeling in the cat auditory nerve. Science 216:1239-1241. CrossRef Medline

Liberman MC, Dodds LW, Pierce S (1990) Afferent and efferent innervation of the cat cochlea: quantitative analysis with light and electron microscopy. J Comp Neurol 301:443-460. CrossRef Medline

Lowen SB, Teich MC (1992) Auditory-nerve action potentials form a nonrenewal point process over short as well as long time scales. J Acoust Soc Am 92:803-806. CrossRef Medline

Meddis R, O'Mard LP (2005) A computer model of the auditory-nerve response to forward-masking stimuli. J Acoust Soc Am 117:3787-3798. CrossRef Medline

Miller MI, Mark KE (1992) A statistical study of cochlear nerve discharge patterns in response to complex speech stimuli. J Acoust Soc Am 92: 202-209. CrossRef Medline

Miller MI, Wang J (1993) A new stochastic model for auditory-nerve discharge. J Acoust Soc Am 94:2093-2107. CrossRef Medline

Moser T, Neef A, Khimich D (2006) Mechanisms underlying the temporal precision of sound coding at the inner hair cell ribbon synapse. J Physiol 576:55-62. CrossRef Medline

Mostafapour SP, Cochran SL, Del Puerto NM, Rubel EW (2000) Patterns of cell death in mouse anteroventral cochlear nucleus neurons after unilateral cochlea removal. J Comp Neurol 426:561-571. CrossRef Medline

Müller M (1991) Developmental changes of frequency representation in the rat cochlea. Hear Res 56:1-7. CrossRef Medline

Müller M (1996) The cochlear place-frequency map of the adult and developing Mongolian gerbil. Hear Res 94:148-156. CrossRef Medline

Peterson AJ, Irvine DR, Heil P (2014) A model of synaptic vesicle-pool depletion and replenishment can account for the interspike interval distributions and nonrenewal properties of spontaneous spike trains of auditory-nerve fibers. J Neurosci 34:15097-15109. CrossRef Medline

Prijs VF, Keijzer J, Versnel H, Schoonhoven R (1993) Recovery characteristics of auditory nerve fibres in the normal and noise-damaged guinea pig cochlea. Hear Res 71:190-201. CrossRef Medline

R Core Team (2014) R: a language and environment for statistical computing. In. Vienna: R Foundation for Statistical Computing. 
Romand R (1984) Functional properties of auditory-nerve fibers during postnatal development in the kitten. Exp Brain Res 56:395-402. Medline

Rutherford MA, Chapochnikov NM, Moser T (2012) Spike encoding of neurotransmitter release timing by spiral ganglion neurons of the cochlea. J Neurosci 32:4773-4789. CrossRef Medline

Sachs MB, Abbas PJ (1974) Rate versus level functions for auditory-nerve fibers in cats: tone-burst stimuli. J Acoust Soc Am 56:1835-1847. CrossRef Medline

Schmiedt RA (1989) Spontaneous rates, thresholds and tuning of auditorynerve fibers in the gerbil: comparisons to cat data. Hear Res 42:23-35. CrossRef Medline

Smolders JW, Klinke R (1984) Effects of temperature on the properties of primary auditory fibers of the spectacled Caiman, Caiman-Crocodilus (L). J Comp Physiol 155:19-30. CrossRef

Stamataki S, Francis HW, Lehar M, May BJ, Ryugo DK (2006) Synaptic alterations at inner hair cells precede spiral ganglion cell loss in aging C57BL/6J mice. Hear Res 221:104-118. CrossRef Medline

Taberner AM, Liberman MC (2005) Response properties of single auditory nerve fibers in the mouse. J Neurophysiol 93:557-569. CrossRef Medline

Teich MC (1989) Fractal character of the auditory neural spike train. IEEE Trans Biomed Eng 36:150-160. CrossRef Medline

Teich MC (1992) Fractal neuronal firing patterns. In: Single neuron computation (McKenna T, Davis J, Zornetzer SF, eds), pp 589-624. San Diego: Academic.

Teich MC, Johnson DH, Kumar AR, Turcott RG (1990) Rate fluctuations and fractional power-law noise recorded from cells in the lower auditory pathway of the cat. Hear Res 46:41-52. CrossRef Medline

Temchin AN, Rich NC, Ruggero MA (2008) Threshold tuning curves of chinchilla auditory nerve fibers: II. Dependence on spontaneous activity and relation to cochlear nonlinearity. J Neurophysiol 100:2899-2906. CrossRef Medline

Terzuolo CA, Araki T (1961) An analysis of intra-versus extracellular potential changes associated with activity of single spinal motoneurons. Ann N Y Acad Sci 94:547-558. CrossRef Medline

Tierney TS, Russell FA, Moore DR (1997) Susceptibility of developing cochlear nucleus neurons to deafferentation-induced death abruptly ends just before the onset of hearing. J Comp Neurol 378:295-306. CrossRef Medline

Tritsch NX, Bergles DE (2010) Developmental regulation of spontaneous activity in the Mammalian cochlea. J Neurosci 30:1539-1550. CrossRef Medline

Tritsch NX, Rodríguez-Contreras A, Crins TT, Wang HC, Borst JG, Bergles DE (2010) Calcium action potentials in hair cells pattern auditory neuron activity before hearing onset. Nat Neurosci 13:1050-1052. CrossRef Medline

Tsuji J, Liberman MC (1997) Intracellular labeling of auditory nerve fibers in guinea pig: central and peripheral projections. J Comp Neurol 381: 188-202. Medline

Uziel A, Romand R, Marot M (1981) Development of cochlear potentials in rats. Audiology 20:89-100. CrossRef Medline

Walsh EJ, McGee J (1987) Postnatal development of auditory nerve and cochlear nucleus neuronal responses in kittens. Hear Res 28:97-116. CrossRef Medline

Wang HC, Bergles DE (2015) Spontaneous activity in the developing auditory system. Cell Tissue Res 361:65-75. CrossRef Medline

Winslow RL, Sachs MB (1988) Single-tone intensity discrimination based on auditory-nerve rate responses in backgrounds of quiet, noise, and with stimulation of the crossed olivocochlear bundle. Hear Res 35:165-189. CrossRef Medline

Wong AB, Jing Z, Rutherford MA, Frank T, Strenzke N, Moser T (2013) Concurrent maturation of inner hair cell synaptic $\mathrm{Ca}^{2+}$ influx and auditory nerve spontaneous activity around hearing onset in mice. J Neurosci 33:10661-10666. CrossRef Medline

Yates GK (1990) Basilar membrane nonlinearity and its influence on auditory nerve rate-intensity functions. Hear Res 50:145-162. CrossRef Medline

Young ED, Barta PE (1986) Rate responses of auditory nerve fibers to tones in noise near masked threshold. J Acoust Soc Am 79:426-442. CrossRef Medline

Young ED, Sachs MB (2008) Auditory nerve inputs to cochlear nucleus neurons studied with cross-correlation. Neuroscience 154:127-138. CrossRef Medline

Youssoufian M, Oleskevich S, Walmsley B (2005) Development of a robust central auditory synapse in congenital deafness. J Neurophysiol 94:3168-3180. CrossRef Medline

Youssoufian M, Couchman K, Shivdasani MN, Paolini AG, Walmsley B (2008) Maturation of auditory brainstem projections and calyces in the congenitally deaf (dn/dn) mouse. J Comp Neurol 506:442-451. CrossRef Medline 